This item was submitted to Loughborough's Research Repository by the author.

Items in Figshare are protected by copyright, with all rights reserved, unless otherwise indicated.

\title{
The inverse spectral transform for the conservative Camassa-Holm flow with decaying initial data
}

PLEASE CITE THE PUBLISHED VERSION

https://doi.org/10.1007/s00205-016-1066-z

\section{PUBLISHER}

(C) the Author. Published by Springer

\section{VERSION}

VoR (Version of Record)

\section{PUBLISHER STATEMENT}

This work is made available according to the conditions of the Creative Commons Attribution 4.0 International (CC BY 4.0) licence. Full details of this licence are available at: http://creativecommons.org/licenses/by/4.0/

\section{LICENCE}

CC BY 4.0

\section{REPOSITORY RECORD}

Eckhardt, Jonathan. 2016. "The Inverse Spectral Transform for the Conservative Camassa-holm Flow with Decaying Initial Data”. Loughborough University. https://hdl.handle.net/2134/35146. 


\title{
The Inverse Spectral Transform for the Conservative Camassa-Holm Flow with Decaying Initial Data
}

\author{
JONATHAN ECKHARDT®
}

Communicated by C. De LeLLIS

\begin{abstract}
We establish the inverse spectral transform for the conservative Camassa-Holm flow with decaying initial data. In particular, it is employed to prove the existence of weak solutions for the corresponding Cauchy problem.
\end{abstract}

\section{Introduction}

The inverse scattering transform $[1,2,39]$ is a powerful tool for solving initial value problems for certain nonlinear partial differential equations. Originally developed for the Korteweg-de Vries equation by Gardner, Greene, Kruskal and MiURA [42], this method has since been successfully extended to various other completely integrable equations. It is the aim of the present article to establish the corresponding transform for the Camassa-Holm equation

$$
u_{t}-u_{x x t}=2 u_{x} u_{x x}-3 u u_{x}+u u_{x x x}
$$

with decaying initial data. Due to the vast amount of literature on this equation, we only refer to a selection of articles $[5,14,15,19,20,26,27,43,50,59,62,63,70]$ containing further information. The relevance of the Camassa-Holm equation stems from the fact that it constitutes a model for unidirectional wave propagation on shallow water $[16,24,52,53]$. Unlike the Korteweg-de Vries equation, it allows for smooth solutions to blow up in finite time in a way that resembles wave-breaking. This process has been described in detail $[20,22,62,63]$ and is known to only happen when the quantity $\omega=u-u_{x x}$ changes sign. Compared to the rather tame sign-definite case (which shares a lot of similarities with the Korteweg-de Vries equation $[4,58,64]$ ), indefiniteness of $\omega$ causes serious complications (for example

Research supported by the Austrian Science Fund (FWF) under Grant No. J3455. 
noticeable from the discussions in $[25,54,57])$. Many of these problems are already apparent for the prototypical example of a peakon-antipeakon collision $[6,20,68]$, which is a special case of so-called multi-peakon solutions [5].

On the other side, the Camassa-Holm equation is known to be formally completely integrable in the sense that there is an associated isospectral problem

$$
-f^{\prime \prime}+\frac{1}{4} f=z \omega f
$$

where $z$ is a complex spectral parameter. Solving the corresponding inverse problem is essentially equivalent to solving the initial value problem for the Camassa-Holm equation. For this reason, it is not surprising that the complications encountered due to wave-breaking for indefinite $\omega$ reoccur when dealing with this inverse problem. In fact, despite a large amount of articles, very little is known about the inverse problem for (1.2) in the indefinite case and almost the entire literature on this subject is restricted to strictly positive and smooth $\omega$ (in which case the spectral problem can be transformed into a standard form that is known from the Kortewegde Vries equation [4,58,64]). Apart from the explicitly solvable finite dimensional case [5,33], only insufficient partial uniqueness results [7,9-11,32,34,37] have been obtained so far for the inverse problem in the indefinite case.

Making use of recent progress on the inverse spectral problem for indefinite strings in [35], we will be able to overcome these difficulties and establish the inverse spectral transform for the more general two-component Camassa-Holm system

$$
\begin{aligned}
u_{t}+u u_{x}+P_{x} & =0, \\
\mu_{t}+(u \mu)_{x} & =\left(u^{3}-2 P u\right)_{x},
\end{aligned}
$$

where the auxiliary function $P$ satisfies

$$
P-P_{x x}=\frac{u^{2}+\mu}{2},
$$

for a class $\mathcal{E}$ of decaying initial data to be defined below; this should be compared to the definition of the set $\mathcal{D}$ in [15, Section 6], [50, Definition 3.1], [45, Definition 4.1]. Note that the two-component Camassa-Holm system (1.3) is equivalent to its more commonly used incarnation in [18,23,40,45,51] (cf. [50, Equation (4.2)]), which is known to contain the Camassa-Holm equation as a special case.

Definition 1.1. The set $\mathcal{D}$ consists of all pairs $(u, \mu)$ such that $u$ is a real-valued function in $H^{1}(\mathbb{R})$ and $\mu$ is a non-negative finite Borel measure on $\mathbb{R}$ with

$$
\mu(B) \geqq \int_{B} u(x)^{2}+u^{\prime}(x)^{2} \mathrm{~d} x
$$

for every Borel set $B \subseteq \mathbb{R}$. 
Associated with each pair $(u, \mu) \in \mathcal{D}$ is a distribution $\omega$ in $H^{-1}(\mathbb{R})$ defined by

$$
\omega(h)=\int_{\mathbb{R}} u(x) h(x) \mathrm{d} x+\int_{\mathbb{R}} u^{\prime}(x) h^{\prime}(x) \mathrm{d} x, \quad h \in H^{1}(\mathbb{R}),
$$

so that $\omega=u-u^{\prime \prime}$ in a distributional sense, as well as a non-negative finite Borel measure $v$ on $\mathbb{R}$ defined such that

$$
\mu(B)=\int_{B} u(x)^{2}+u^{\prime}(x)^{2} \mathrm{~d} x+v(B)
$$

for every Borel set $B \subseteq \mathbb{R}$. Let us point out that it is always possible to uniquely recover the pair $(u, \mu)$ from the distribution $\omega$ and the Borel measure $v$. In fact, the function $u$ at any point $x \in \mathbb{R}$ can be written as

$$
u(x)=\omega\left(\delta_{x}\right), \quad \delta_{x}(s)=\frac{1}{2} \mathrm{e}^{-|x-s|}, \quad s \in \mathbb{R},
$$

which then allows us to determine the Borel measure $\mu$ as well from (1.7). Now the class $\mathcal{E}$ is defined by imposing an additional growth restriction on pairs in $\mathcal{D}$.

Definition 1.2. The set $\mathcal{E}_{ \pm}$consists of all pairs $(u, \mu) \in \mathcal{D}$ such that

$$
\int_{\mathbb{R}} \mathrm{e}^{ \pm x}\left(u^{\prime}(x) \mp u(x)\right)^{2} \mathrm{~d} x+\int_{\mathbb{R}} \mathrm{e}^{ \pm x} \mathrm{~d} v(x)<\infty .
$$

Furthermore, the set $\mathcal{E}$ is defined as the intersection of $\mathcal{E}_{+}$and $\mathcal{E}_{-}$.

Justified by the Lax pair formulation of the two-component Camassa-Holm system in $[18,23,51]$ and the results in [33], we will consider the spectral problem

$$
-f^{\prime \prime}+\frac{1}{4} f=z \omega f+z^{2} v f
$$

Due to the low regularity of the coefficients, it is not clear how this differential equation has to be interpreted, which is why we clarify this matter in Appendix A. Basic properties of the spectral problem (like realness and discreteness of the spectrum $\sigma$ for example) will be discussed in Section 2 along with some further necessary conditions. In the following section, we will solve the corresponding inverse problem for the class of coefficients corresponding to $\mathcal{E}$, giving a complete characterization of all possible spectral data. More precisely, we will establish a one-to-one correspondence between $\mathcal{E}$ and a class of spectral data explicitly described by Theorem 3.1, which will be shown to be a homeomorphism with respect to suitable topologies. Finally, in Section 4 we will introduce the conservative Camassa-Holm flow as a certain isospectral flow on $\mathcal{E}$ and show that its integral curves define weak solutions of the two-component Camassa-Holm system. 
As an immediate consequence of the solution of the inverse spectral problem in Theorem 3.1, we will see that the phase space $\mathcal{E}$ decomposes into a foliation of invariant isospectral sets Iso $(\sigma)$, each of which can be parametrized by the $\operatorname{set}^{1}$

$$
\left\{\kappa \in \mathbb{R}^{\sigma} \mid \sum_{\lambda \in \sigma} \frac{1}{\lambda^{2}} \frac{\mathrm{e}^{\left|\kappa_{\lambda}\right|}}{|\lambda \dot{W}(\lambda)|}<\infty\right\},
$$

where $W$ is the entire function defined by the infinite product in (1.16) and the dot denotes differentiation. In terms of these coordinates, the conservative CamassaHolm flow on each set Iso $(\sigma)$ becomes the simple linear flow given by

$$
\kappa_{\lambda}^{\prime}=\frac{1}{2 \lambda}, \quad \lambda \in \sigma
$$

These facts are reminiscent of the fact that the conservative Camassa-Holm flow can be viewed as a completely integrable infinite dimensional Hamiltonian system.

Notation. For integrals of a function $f$ which is locally integrable with respect to a Borel measure $v$ on an interval $I$, we will employ the convenient notation

$$
\int_{x}^{y} f \mathrm{~d} \nu= \begin{cases}\int_{[x, y)} f \mathrm{~d} \nu, & y>x, \\ 0, & y=x, \quad x, y \in I, \\ -\int_{[y, x)} f \mathrm{~d} \nu, & y<x,\end{cases}
$$

rendering the integral left-continuous as a function of $y$. If the function $f$ is locally absolutely continuous on $I$ and $g$ denotes a left-continuous distribution function of the Borel measure $v$, then we have the integration by parts formula

$$
\int_{x}^{y} f \mathrm{~d} v=\left.g f\right|_{x} ^{y}-\int_{x}^{y} g(s) f^{\prime}(s) \mathrm{d} s, \quad x, y \in I,
$$

which will be used frequently throughout this article.

Given a discrete set $\sigma$ of nonzero reals, we denote with $n_{\sigma}(r)$ the number of all $\lambda \in \sigma$ with modulus not greater than $r$. Furthermore, we introduce the notation

$$
\sum_{\lambda \in \sigma}^{\leftrightarrow} \frac{1}{\lambda}=\lim _{r \rightarrow \infty} \sum_{\substack{\lambda \in \sigma \\|\lambda| \leqq r}} \frac{1}{\lambda}
$$

provided that the limit exists. Similarly, subject to existence, we shall write

$$
\prod_{\lambda \in \sigma}^{\leftrightarrow}\left(1-\frac{z}{\lambda}\right)=\lim _{r \rightarrow \infty} \prod_{\substack{\lambda \in \sigma \\|\lambda| \leqq r}}\left(1-\frac{z}{\lambda}\right), \quad z \in \mathbb{C},
$$

where the limit is meant to be taken in the topology of locally uniform convergence. The limit in (1.16) exists if and only if the limit in (1.15) exists and the sum

${ }^{1}$ We denote with $\mathbb{R}^{\sigma}$ the set of all real-valued sequences $\kappa=\left\{\kappa_{\lambda}\right\}_{\lambda \in \sigma}$ indexed by $\sigma$. 


$$
\sum_{\lambda \in \sigma} \frac{1}{\lambda^{2}}
$$

is finite. In this case, upon denoting the entire function in (1.16) with $W$, we have

$$
\sum_{\lambda \in \sigma}^{\leftrightarrow} \frac{1}{\lambda}=-\dot{W}(0), \quad \quad \quad \sum_{\lambda \in \sigma} \frac{1}{\lambda^{2}}=\dot{W}(0)^{2}-\ddot{W}(0) .
$$

\section{The Direct Spectral Problem}

In the present section, we are going to introduce the spectral quantities that will linearize the conservative Camassa-Holm flow on $\mathcal{E}$ and derive their basic properties. To this end, we fix an arbitrary pair $(u, \mu) \in \mathcal{E}$ and first recall the definition of the distribution $\omega$ in (1.6) as well as the one of the Borel measure $v$ in (1.7). We introduce the spectrum $\sigma$ associated with the pair $(u, \mu)$ as the set of all those numbers $z \in \mathbb{C}$ for which there is a nontrivial solution $f$ of the differential equation

$$
-f^{\prime \prime}+\frac{1}{4} f=z \omega f+z^{2} v f
$$

that belongs to $H^{1}(\mathbb{R})$. Regarding the precise meaning and some basic properties of this differential equation we refer to the discussion in Appendix A.

A first consequence of the growth restriction on $(u, \mu)$ in (1.9) is the existence of particular solutions of the differential equation (2.1) with prescribed asymptotics.

Theorem 2.1. For every $z \in \mathbb{C}$ there is a unique solution $\phi_{ \pm}(z, \cdot)$ of the differential equation (2.1) with the asymptotics

$$
\phi_{ \pm}(z, x) \sim \mathrm{e}^{\mp \frac{x}{2}}, \quad x \rightarrow \pm \infty .
$$

The derivative of $\phi_{ \pm}(z, \cdot)$ is integrable and square integrable near $\pm \infty$.

Proof. To begin with, let us introduce the diffeomorphism $\eta_{ \pm}: \mathbb{R}_{\mp} \rightarrow \mathbb{R}$ by

$$
\eta_{ \pm}(\xi)=\mp \ln (\mp \xi), \quad \xi \in \mathbb{R}_{\mp},
$$

where $\mathbb{R}_{\mp}$ denotes the open negative/positive semi-axis. Furthermore, let $\mathrm{a}_{ \pm}$be a measurable function on $\mathbb{R}_{\mp}$ such that (we set $\alpha_{ \pm}=-u^{\prime} \pm u$ as in Appendix A)

$$
\mathrm{a}_{ \pm}(\xi)=\frac{\alpha_{ \pm}\left(\eta_{ \pm}(\xi)\right)}{\mp \xi}=\frac{-u^{\prime}\left(\eta_{ \pm}(\xi)\right) \pm u\left(\eta_{ \pm}(\xi)\right)}{\mp \xi}
$$

for almost all $\xi \in \mathbb{R}_{\mp}$ (note that the right-hand side is well-defined almost everywhere) and define the non-negative Borel measure $\beta_{ \pm}$on $\mathbb{R}_{\mp}$ via setting

$$
\beta_{ \pm}(B)=\int_{B} \frac{1}{\mp \xi} \mathrm{d} v \circ \eta_{ \pm}(\xi)=\int_{\eta_{ \pm}(B)} \mathrm{e}^{ \pm x} \mathrm{~d} v(x)
$$


for every Borel set $B \subseteq \mathbb{R}_{\mp}$. The growth restriction on $(u, \mu)$ in (1.9) implies that the function $\mathrm{a}_{ \pm}$is square integrable and that the measure $\beta_{ \pm}$is finite (thus $\beta_{ \pm}$can be extended to a Borel measure on the closure of $\mathbb{R}_{\mp}$ by setting $\left.\beta_{ \pm}(\{0\})=0\right)$. Consequently (see, for example, [3, Section 11.8], [8, Theorem 1.1], [38, Theorem A.2]), there is a unique matrix solution $Y_{ \pm}(z, \cdot)$ on $\mathbb{R}_{\mp}$ of the integral equation

$$
\begin{aligned}
Y_{ \pm}(z, \xi)= & \left(\begin{array}{ll}
1 & 0 \\
0 & 1
\end{array}\right)+z \int_{0}^{\xi}\left(\begin{array}{cc}
-\mathrm{a}_{ \pm}(s) & -1 \\
\mathrm{a}_{ \pm}(s)^{2} & \mathrm{a}_{ \pm}(s)
\end{array}\right) Y_{ \pm}(z, s) \mathrm{d} s \\
& +z \int_{0}^{\xi}\left(\begin{array}{ll}
0 & 0 \\
1 & 0
\end{array}\right) Y_{ \pm}(z, s) \mathrm{d} \beta_{ \pm}(s), \quad \xi \in \mathbb{R}_{\mp},
\end{aligned}
$$

for each $z \in \mathbb{C}$. It follows from a version of Gronwall's inequality (see, for example, [8, Lemma 1.3], [38, Lemma A.1]) that the function $Y_{ \pm}(z, \cdot)$ satisfies the estimate

$$
\left\|Y_{ \pm}(z, \xi)\right\| \leqq \mathrm{e}^{\Lambda_{ \pm}(\xi)|z|}, \quad \xi \in \mathbb{R}_{\mp},
$$

where $\|\cdot\|$ denotes the max norm and the non-negative function $\Lambda_{ \pm}$is given by

$$
\mp \Lambda_{ \pm}(\xi)=2 \int_{0}^{\xi} \max \left(1, \mathrm{a}_{ \pm}(s)^{2}\right) \mathrm{d} s+\int_{0}^{\xi} \mathrm{d} \beta_{ \pm}, \quad \xi \in \mathbb{R}_{\mp} .
$$

Plugging this estimate back into the integral equation (2.5), we furthermore get

$$
\left\|Y_{ \pm}(z, \xi)-\left(\begin{array}{ll}
1 & 0 \\
0 & 1
\end{array}\right)\right\| \leqq|z| \Lambda_{ \pm}(\xi) \mathrm{e}^{\Lambda_{ \pm}(\xi)|z|}, \quad \xi \in \mathbb{R}_{\mp} .
$$

For the top-right entry of $Y_{ \pm}(z, \cdot)$, we are actually able to sharpen the estimate to

$$
\left|Y_{ \pm, 12}(z, \xi)\right| \leqq|z| \mathrm{e}^{\Lambda_{ \pm}(\xi)|z|}\left(\mp \int_{0}^{\xi}\left|\mathrm{a}_{ \pm}(s)\right| \mathrm{d} s \mp \xi\right), \quad \xi \in \mathbb{R}_{\mp} .
$$

Upon plugging this back into the integral equation (2.5) one more time, we obtain

$$
\left|Y_{ \pm, 12}(z, \xi)+z \xi\right| \leqq 2|z|^{2}|\xi| \Lambda_{ \pm}(\xi) \mathrm{e}^{\Lambda_{ \pm}(\xi)|z|}, \quad \xi \in \mathbb{R}_{\mp}
$$

Next, we introduce the matrix function $U_{ \pm}(z, \cdot)$ on $\mathbb{R}$ by

$$
U_{ \pm}(z, x)=\left(\begin{array}{cc}
\mathrm{e}^{ \pm \frac{x}{2}} & 0 \\
0 & \mathrm{e}^{\mp \frac{x}{2}}
\end{array}\right) Y_{ \pm}\left(z, \eta_{ \pm}^{-1}(x)\right), \quad x \in \mathbb{R} .
$$

From the integral equation (2.5), a substitution (use, for example, [13, Theorem 3.6.1 and Corollary 3.7.2]) as well as the definitions of $a_{ \pm}$and $\beta_{ \pm}$, one sees that

$$
\begin{aligned}
\left.Y_{ \pm}\left(z, \eta_{ \pm}^{-1}(\cdot)\right)\right|_{x} ^{y}= & z \int_{x}^{y}\left(\begin{array}{cc}
-\alpha_{ \pm}(s) & -\mathrm{e}^{\mp s} \\
\alpha_{ \pm}(s)^{2} \mathrm{e}^{ \pm s} & \alpha_{ \pm}(s)
\end{array}\right) Y_{ \pm}\left(z, \eta_{ \pm}^{-1}(s)\right) \mathrm{d} s \\
& +z \int_{x}^{y}\left(\begin{array}{cc}
0 & 0 \\
\mathrm{e}^{ \pm s} & 0
\end{array}\right) Y_{ \pm}\left(z, \eta_{ \pm}^{-1}(s)\right) \mathrm{d} v(s)
\end{aligned}
$$

for all $x, y \in \mathbb{R}$. Upon employing the integration by parts formula (1.14), one sees that $U_{ \pm}(z, \cdot)$ is a solution of the integral equation (A.5) and thus the system (A.4). 
Now under the additional assumption that $z$ is non-zero, let $\theta_{ \pm}(z, \cdot)$ and $\phi_{ \pm}(z, \cdot)$ be the solutions of the differential equation (2.1) such that

$$
U_{ \pm}(z, x)= \pm\left(\begin{array}{cc}
-\theta_{ \pm}(z, x) & z \phi_{ \pm}(z, x) \\
\frac{1}{z} \theta_{ \pm}^{[ \pm]}(z, x) & -\phi_{ \pm}^{[ \pm]}(z, x)
\end{array}\right), \quad x \in \mathbb{R}
$$

guaranteed to exist by Lemma A.2. For future purposes, let us note the bounds

$$
\begin{aligned}
& \left|\theta_{ \pm}(z, x) \mathrm{e}^{\mp \frac{x}{2}}\right|,\left|\theta_{ \pm}^{[1]}(z, x) \mathrm{e}^{\mp \frac{x}{2}}\right|,\left|\phi_{ \pm}(z, x) \mathrm{e}^{ \pm \frac{x}{2}}\right|,\left|\phi_{ \pm}^{[1]}(z, x) \mathrm{e}^{ \pm \frac{x}{2}}\right| \\
& \quad \leq 2 \mathrm{e}^{4 \Lambda_{ \pm}\left(\mp \mathrm{e}^{\mp x}\right)|z|}, \quad x \in \mathbb{R},
\end{aligned}
$$

that follow from the estimates (2.6) and (2.8) for $Y_{ \pm}(z, \cdot)$, also using that

$$
|u(x)|=\left|\int_{x}^{ \pm \infty} \mathrm{e}^{-|x-s|} \alpha_{ \pm}(s) \mathrm{d} s\right| \leqq \frac{1}{2} \Lambda_{ \pm}\left(\mp \mathrm{e}^{\mp x}\right), \quad x \in \mathbb{R} .
$$

Furthermore, the inequalities in (2.7) and (2.8) turn into

$$
\begin{aligned}
& \left|\theta_{ \pm}(z, x) \mathrm{e}^{\mp \frac{x}{2}} \pm 1\right|,\left|\frac{1}{z} \theta_{ \pm}^{[ \pm]}(z, x) \mathrm{e}^{ \pm \frac{x}{2}}\right|,\left|\phi_{ \pm}(z, x) \mathrm{e}^{ \pm \frac{x}{2}}-1\right|,\left|\phi_{ \pm}^{[ \pm]}(z, x) \mathrm{e}^{ \pm \frac{x}{2}} \pm 1\right| \\
& \quad \leqq 2|z| \Lambda_{ \pm}\left(\mp \mathrm{e}^{\mp x}\right) \mathrm{e}^{\Lambda_{ \pm}\left(\mp \mathrm{e}^{\mp x}\right)|z|}, \quad x \in \mathbb{R} .
\end{aligned}
$$

In particular, we see that $\phi_{ \pm}(z, \cdot)$ has the required asymptotics (2.2) as well as

$$
\theta_{ \pm}(z, x) \sim \mp \mathrm{e}^{ \pm \frac{x}{2}}, \quad x \rightarrow \pm \infty,
$$

which implies that $\phi_{ \pm}(z, \cdot)$ is indeed uniquely determined by the asymptotics in (2.2). Finally, the fact that the derivative of $\phi_{ \pm}(z, \cdot)$ is integrable and square integrable near $\pm \infty$ follows from the last bound in (2.9). It remains to set

$$
\phi_{ \pm}(0, x)=\mathrm{e}^{\mp \frac{x}{2}}, \quad \theta_{ \pm}(0, x)=\mp \mathrm{e}^{ \pm \frac{x}{2}},
$$

for all $x \in \mathbb{R}$ and note that the claim is obvious in the case when $z$ is zero.

For every $z \in \mathbb{C}$ we introduce the complex number $W(z)$ as the Wronskian of the two solutions $\phi_{+}(z, \cdot)$ and $\phi_{-}(z, \cdot)$, that is, in such a way that

$$
W(z)=\phi_{+}(z, x) \phi_{-}^{\prime}(z, x)-\phi_{+}^{\prime}(z, x) \phi_{-}(z, x)
$$

for almost all $x \in \mathbb{R}$; see Corollary A.4. It follows readily that the set of zeros of $W$ coincides with the spectrum $\sigma$. Thus, the next result implies that $\sigma$ is a discrete set of nonzero reals with a convergence exponent of at most one.

Corollary 2.2. The functions $\phi_{ \pm}(\cdot, x)$ and $W$ are real entire of Cartwright class with only nonzero and real roots for each fixed $x \in \mathbb{R}$. Moreover, the function

$$
\frac{z \phi_{-}(z, x) \phi_{+}(z, x)}{W(z)}, \quad z \in \mathbb{C} \backslash \mathbb{R},
$$

is a meromorphic Herglotz-Nevanlinna function. 
Proof. Since the entries of $Y_{ \pm}(\cdot, \xi)$ are real entire of Cartwright class with only real roots (cf. [55, Section 1]), the same holds for $\phi_{ \pm}(\cdot, x)$ upon noting that

$$
\lim _{z \rightarrow 0} \phi_{ \pm}(z, x)= \pm \mathrm{e}^{ \pm \frac{x}{2}} \lim _{z \rightarrow 0} \frac{Y_{ \pm, 12}\left(z, \mp \mathrm{e}^{\mp x}\right)}{z}=\mathrm{e}^{\mp \frac{x}{2}}=\phi_{ \pm}(0, x) .
$$

Because the function $\phi_{ \pm}^{[1]}(\cdot, x)$ is real entire of Cartwright class as well, so is $W$ as

$$
W(z)=\phi_{+}(z, x) \phi_{-}^{[1]}(z, x)-\phi_{+}^{[1]}(z, x) \phi_{-}(z, x), \quad z \in \mathbb{C} .
$$

Next, we observe that the meromorphic function

$$
\pm \frac{\phi_{ \pm}^{[1]}(z, x)}{z \phi_{ \pm}(z, x)}, \quad z \in \mathbb{C} \backslash \mathbb{R}
$$

is a Herglotz-Nevanlinna function. In fact, to this end one just needs to evaluate its imaginary part and use (A.12) as well as the vanishing asymptotics of the solution $\phi_{ \pm}(z, \cdot)$ near $\pm \infty$. Since we may write

$$
-\frac{W(z)}{z \phi_{-}(z, x) \phi_{+}(z, x)}=\frac{\phi_{+}^{[1]}(z, x)}{z \phi_{+}(z, x)}-\frac{\phi_{-}^{[1]}(z, x)}{z \phi_{-}(z, x)}, \quad z \in \mathbb{C} \backslash \mathbb{R},
$$

this shows that the function in (2.12) is a Herglotz-Nevanlinna function as well. In particular, this guarantees that $W$ has only nonzero and real roots indeed.

Remark 2.3. Although we will not prove this here, let us mention that it is possible to show that the exponential type of the entire function $W$ is simply given by

$$
\int_{\mathbb{R}} \rho(x) \mathrm{d} x,
$$

where $\rho$ is the square root of the Radon-Nikodým derivative of the absolutely continuous part of the Borel measure $v$ (with respect to the Lebesgue measure).

As the spectrum alone will not be enough, we need to introduce further spectral quantities. Since the solutions $\phi_{+}(\lambda, \cdot)$ and $\phi_{-}(\lambda, \cdot)$ are linearly dependent for each fixed $\lambda \in \sigma$ in view of Corollary A.4, there is a unique nonzero $c_{\lambda} \in \mathbb{R}$ such that

$$
\phi_{-}(\lambda, x)=c_{\lambda} \phi_{+}(\lambda, x), \quad x \in \mathbb{R},
$$

which will be referred to as the coupling constant associated with $\lambda$. It will often be more convenient to work with the logarithmic coupling constant $\kappa_{\lambda}$, defined by

$$
\kappa_{\lambda}=\ln \left|c_{\lambda}\right|
$$

instead. We will see below that one can always recover (the sign of) the coupling constant $c_{\lambda}$ from the quantity $\kappa_{\lambda}$, provided the spectrum $\sigma$ is known as well. Furthermore, we introduce the right/left (modified) norming constant $\gamma_{\lambda, \pm}^{2} \in \mathbb{R}$ via

$$
\gamma_{\lambda, \pm}^{2}=\omega\left(\phi_{ \pm}(\lambda, \cdot)^{2}\right)+2 \lambda \int_{\mathbb{R}} \phi_{ \pm}(\lambda, x)^{2} \mathrm{~d} v(x) .
$$


Upon employing (A.12) and the asymptotics from Theorem 2.1, one sees that

$$
\lambda \gamma_{\lambda, \pm}^{2}=\int_{\mathbb{R}} \phi_{ \pm}^{\prime}(\lambda, x)^{2} \mathrm{~d} x+\frac{1}{4} \int_{\mathbb{R}} \phi_{ \pm}(\lambda, x)^{2} \mathrm{~d} x+\lambda^{2} \int_{\mathbb{R}} \phi_{ \pm}(\lambda, x)^{2} \mathrm{~d} v(x)>0 .
$$

The following result gives a relation between all our spectral quantities.

Lemma 2.4. For each $\lambda \in \sigma$ we have the relation

$$
-\dot{W}(\lambda)=c_{\lambda}^{ \pm 1} \gamma_{\lambda, \pm}^{2}
$$

Proof. Let us fix an arbitrary $z \in \mathbb{C}$ and introduce the function

$$
W_{ \pm}(z, x)=\dot{\phi}_{ \pm}(z, x) \phi_{\mp}^{[1]}(z, x)-\dot{\phi}_{ \pm}^{[1]}(z, x) \phi_{\mp}(z, x), \quad x \in \mathbb{R},
$$

where the dot denotes differentiation with respect to the spectral parameter. From the bounds in (2.10) and Cauchy's integral formula, we obtain the estimates

$$
\begin{aligned}
& \left|\dot{\phi}_{ \pm}(z, x) \mathrm{e}^{ \pm \frac{x}{2}}\right|,\left|\dot{\phi}_{ \pm}^{[ \pm]}(z, x) \mathrm{e}^{ \pm \frac{x}{2}}\right| \\
& \quad \leqq 2(|z|+1) \Lambda_{ \pm}\left(\mp \mathrm{e}^{\mp x}\right) \mathrm{e}^{\Lambda_{ \pm}\left(\mp \mathrm{e}^{\mp x}\right)(|z|+1)}, \quad x \in \mathbb{R} .
\end{aligned}
$$

Upon writing $\phi_{\mp}(z, \cdot)$ as a linear combination of $\phi_{ \pm}(z, \cdot)$ and $\theta_{ \pm}(z, \cdot)$, we see from the bounds in (2.9) that the function $W_{ \pm}(z, x)$ tends to zero as $x \rightarrow \pm \infty$. Next, we note that from (A.6) and (A.8) one gets

$$
\begin{aligned}
\left.\phi_{ \pm}(z, \cdot)\right|_{x} ^{y} & =\int_{x}^{y} z u^{\prime}(s) \phi_{ \pm}(z, s)+\phi_{ \pm}^{[1]}(z, s) \mathrm{d} s \\
\left.\phi_{ \pm}^{[1]}(z, \cdot)\right|_{x} ^{y}= & \int_{x}^{y}\left(\frac{1}{4}-z u(s)-z^{2} u^{\prime}(s)^{2}\right) \phi_{ \pm}(z, s)-z u^{\prime}(s) \phi_{ \pm}^{[1]}(z, s) \mathrm{d} s \\
& -z^{2} \int_{x}^{y} \phi_{ \pm}(z, s) \mathrm{d} v(s)
\end{aligned}
$$

for all $x, y \in \mathbb{R}$, and after differentiating with respect to $z$ also

$$
\begin{aligned}
\left.\dot{\phi}_{ \pm}(z, \cdot)\right|_{x} ^{y}= & \int_{x}^{y} z u^{\prime}(s) \dot{\phi}_{ \pm}(z, s)+\dot{\phi}_{ \pm}^{[1]}(z, s)+u^{\prime}(s) \phi_{ \pm}(z, s) \mathrm{d} s \\
\left.\dot{\phi}_{ \pm}^{[1]}(z, \cdot)\right|_{x} ^{y}= & \int_{x}^{y}\left(\frac{1}{4}-z u(s)-z^{2} u^{\prime}(s)^{2}\right) \dot{\phi}_{ \pm}(z, s)-z u^{\prime}(s) \dot{\phi}_{ \pm}^{[1]}(z, s) \mathrm{d} s \\
& -\int_{x}^{y}\left(u(s)+2 z u^{\prime}(s)^{2}\right) \phi_{ \pm}(z, s)+u^{\prime}(s) \phi_{ \pm}^{[1]}(z, s) \mathrm{d} s \\
& -2 z \int_{x}^{y} \phi_{ \pm}(z, s) \mathrm{d} v(s)-z^{2} \int_{x}^{y} \dot{\phi}_{ \pm}(z, s) \mathrm{d} v(s) .
\end{aligned}
$$

In conjunction with the integration by parts formula (1.14), this gives

$$
\begin{aligned}
\left.W_{ \pm}(z, \cdot)\right|_{x} ^{y}= & \int_{x}^{y} u(s) \phi_{+}(z, s) \phi_{-}(z, s)+u^{\prime}(s)\left(\phi_{+}(z, \cdot) \phi_{-}(z, \cdot)\right)^{\prime}(s) \mathrm{d} s \\
& +2 z \int_{x}^{y} \phi_{+}(z, s) \phi_{-}(z, s) \mathrm{d} v(s) .
\end{aligned}
$$


Upon letting $y \rightarrow \pm \infty$ in this equation, we obtain

$$
\begin{aligned}
-\dot{W}(z)= & W_{-}(z, x)-W_{+}(z, x) \\
= & \int_{\mathbb{R}} u(s) \phi_{+}(z, s) \phi_{-}(z, s)+u^{\prime}(s)\left(\phi_{+}(z, \cdot) \phi_{-}(z, \cdot)\right)^{\prime}(s) \mathrm{d} s \\
& +2 z \int_{\mathbb{R}} \phi_{+}(z, s) \phi_{-}(z, s) \mathrm{d} v(s),
\end{aligned}
$$

which readily yields the claimed identity (one should also note that the functions $u$ and $u^{\prime}$ are integrable due to the growth restriction in (1.9) and thus the bounds in (2.9) guarantee that all integrals exist indeed).

In particular, the previous result shows that all zeros of the entire function $W$ are simple. As a Cartwright class function, the Wronskian $W$ thus admits a product representation (see, for example, [60, Section 17.2]) of the form

$$
W(z)=\prod_{\lambda \in \sigma}^{\leftrightarrow}\left(1-\frac{z}{\lambda}\right), \quad z \in \mathbb{C}
$$

Upon invoking the identities in (1.18), this fact allows us to read off trace formulas for the spectrum $\sigma$ from the derivatives of the function $W$ at zero.

Proposition 2.5. The first two trace formulas are:

$$
\sum_{\lambda \in \sigma}^{\leftrightarrow} \frac{1}{\lambda}=\int_{\mathbb{R}} u(x) d x, \quad \frac{1}{2} \sum_{\lambda \in \sigma} \frac{1}{\lambda^{2}}=\int_{\mathbb{R}} d \mu .
$$

Proof. In view of (1.18), we immediately obtain the first identity from (2.19) and we are left to compute the second derivative of $W$ at zero. For this purpose, we first introduce the entire function $V_{ \pm}$by

$$
V_{ \pm}(z)=\theta_{ \pm}(z, x) \phi_{\mp}^{[1]}(z, x)-\theta_{ \pm}^{[1]}(z, x) \phi_{\mp}(z, x), \quad x \in \mathbb{R}, z \in \mathbb{C},
$$

so that we may write

$$
\phi_{ \pm}(z, x)= \pm W(z) \theta_{\mp}(z, x)+V_{\mp}(z) \phi_{\mp}(z, x), \quad x \in \mathbb{R}, z \in \mathbb{C} .
$$

In conjunction with (2.9) and (2.10), this allows us to estimate

$$
\begin{aligned}
& \left|\phi_{ \pm}(z, x) \mathrm{e}^{ \pm \frac{x}{2}}-W(z)\right|,\left|\phi_{ \pm}^{[ \pm]}(z, x) \mathrm{e}^{ \pm \frac{x}{2}} \pm W(z)\right| \\
& \quad \leqq 4 \mathrm{e}^{5 \Lambda_{\mp}\left( \pm \mathrm{e}^{ \pm x}\right)|z|}\left(|z W(z)| \Lambda_{\mp}\left( \pm \mathrm{e}^{ \pm x}\right)\left(|z| \mathrm{e}^{ \pm x}+1\right)+\left|V_{\mp}(z)\right| \mathrm{e}^{ \pm x}\right)
\end{aligned}
$$

for all $x \in \mathbb{R}$ and $z \in \mathbb{C}$. From this we see that each of the three products

$$
\phi_{+}(z, x) \phi_{-}(z, x), \quad \phi_{+}(z, x) \phi_{-}^{[1]}(z, x), \quad \phi_{+}^{[1]}(z, x) \phi_{-}(z, x),
$$


is bounded uniformly in $x \in \mathbb{R}$ and locally uniformly in $z \in \mathbb{C}$. Thus, we may differentiate (2.19) under the integral and in order to evaluate the derivative at zero, we first note that

$$
\dot{Y}_{ \pm}(0, \xi)=\int_{0}^{\xi}\left(\begin{array}{cc}
-\mathrm{a}_{ \pm}(s) & -1 \\
\mathrm{a}_{ \pm}(s)^{2} & \mathrm{a}_{ \pm}(s)
\end{array}\right) \mathrm{d} s+\int_{0}^{\xi}\left(\begin{array}{ll}
0 & 0 \\
1 & 0
\end{array}\right) \mathrm{d} \beta_{ \pm}(s), \quad \xi \in \mathbb{R}_{\mp},
$$

which follows from (2.5). Moreover, for the top-right entry we even have

$$
\ddot{Y}_{ \pm, 12}(0, \xi)=2 \xi \int_{0}^{\xi} \mathrm{a}_{ \pm}(s) \mathrm{d} s-4 \int_{0}^{\xi} \int_{0}^{s} \mathrm{a}_{ \pm}(r) \mathrm{d} r \mathrm{~d} s, \quad \xi \in \mathbb{R}_{\mp} .
$$

After performing substitutions, this turns into the identities

$$
\dot{\phi}_{ \pm}(0, x)=\mathrm{e}^{\mp \frac{x}{2}} \int_{x}^{ \pm \infty} \alpha_{\mp}(s) \mathrm{d} s, \quad \dot{\phi}_{ \pm}^{[1]}(0, x)= \pm \frac{1}{2} \mathrm{e}^{\mp \frac{x}{2}} \int_{x}^{ \pm \infty} \alpha_{ \pm}(s) \mathrm{d} s,
$$

for all $x \in \mathbb{R}$. Plugging them into the differentiated integral in (2.19), we arrive at

$$
-\ddot{W}(0)=2 \int_{\mathbb{R}} u(x)^{2}+u^{\prime}(x)^{2} \mathrm{~d} x+2 \int_{\mathbb{R}} \mathrm{d} v-\left(\int_{\mathbb{R}} u(x) \mathrm{d} x\right)^{2},
$$

which proves the remaining claim in view of (1.18).

Before we are able to solve the inverse spectral problem, we need to derive one more necessary condition. More precisely, we will show that the growth restriction on the pair $(u, \mu)$ in (1.9) implies certain asymptotic behavior of the spectral data.

Proposition 2.6. We have the identity

$$
\int_{\mathbb{R}} \mathrm{e}^{ \pm x}\left(u^{\prime}(x) \mp u(x)\right)^{2} \mathrm{~d} x+\int_{\mathbb{R}} \mathrm{e}^{ \pm x} \mathrm{~d} v(x)=\sum_{\lambda \in \sigma} \frac{1}{\lambda^{2}} \frac{1}{\lambda \gamma_{\lambda, \pm}^{2}} .
$$

Proof. To begin with, we introduce the function $m_{ \pm}$by

$$
m_{ \pm}(z)=\frac{V_{ \pm}(z)}{z W(z)}, \quad z \in \mathbb{C} \backslash \mathbb{R} .
$$

From the estimates in (2.23), we see that

$$
\phi_{ \pm}(z, x) \mathrm{e}^{ \pm \frac{x}{2}} \rightarrow W(z), \quad \phi_{ \pm}^{[ \pm]}(z, x) \mathrm{e}^{ \pm \frac{x}{2}} \rightarrow \mp W(z), \quad x \rightarrow \mp \infty,
$$

where the convergence is locally uniform in $z \in \mathbb{C}$. In much the same manner as above (upon writing $\theta_{ \pm}$as in (2.22) and using (2.9) as well as (2.10) to estimate), one obtains inequalities similar to (2.23) for $\theta_{ \pm}$and concludes that

$$
\theta_{ \pm}(z, x) \mathrm{e}^{ \pm \frac{x}{2}} \rightarrow \pm V_{ \pm}(z), \quad \theta_{ \pm}^{[ \pm]}(z, x) \mathrm{e}^{ \pm \frac{x}{2}} \rightarrow-V_{ \pm}(z), \quad x \rightarrow \mp \infty,
$$

where the convergence is again locally uniform in $z \in \mathbb{C}$. Thus, we may write

$$
m_{ \pm}(z)= \pm \lim _{x \rightarrow \mp \infty} \frac{\theta_{ \pm}(z, x)}{z \phi_{ \pm}(z, x)}=\mp \lim _{\xi \rightarrow \mp \infty} \frac{Y_{ \pm, 11}(z, \xi)}{Y_{ \pm, 12}(z, \xi)}, \quad z \in \mathbb{C} \backslash \mathbb{R}
$$


that is, the function $m_{ \pm}$is the Weyl-Titchmarsh function for (2.5); cf. [35, Equation (6.2)]. In particular, it is a meromorphic Herglotz-Nevanlinna function [35, Lemma 5.1] with simple poles at all points $\lambda \in \sigma$ (note that zero is not a pole since $V_{ \pm}$vanishes at zero) with residues given by

$$
\operatorname{res}_{\lambda} m_{ \pm}=\frac{V_{ \pm}(\lambda)}{\lambda \dot{W}(\lambda)}=-\frac{1}{\lambda \gamma_{\lambda, \pm}^{2}}, \quad \lambda \in \sigma,
$$

in view of Lemma 2.4. After a substitution, we obtain from (2.24) that

$$
\dot{\theta}_{ \pm}(0, x)=\mp \mathrm{e}^{ \pm \frac{x}{2}} \int_{x}^{ \pm \infty} \alpha_{ \pm}(s) \mathrm{d} s, \quad \dot{\theta}_{ \pm}^{[1]}(0, x)=\frac{1}{2} \mathrm{e}^{ \pm \frac{x}{2}} \int_{x}^{ \pm \infty} \alpha_{\mp}(s) \mathrm{d} s,
$$

for every $x \in \mathbb{R}$, which implies that $\dot{V}_{ \pm}(0)=0$. Upon recalling the integral representation formula for Herglotz-Nevanlinna functions (see, for example, [66, Section 5.3]), this guarantees that the function $m_{ \pm}$admits the representation (use also [35, Lemma 7.1] to conclude that there is no linear term present)

$$
m_{ \pm}(z)=\sum_{\lambda \in \sigma} \frac{z}{\lambda(\lambda-z)} \frac{1}{\lambda \gamma_{\lambda, \pm}^{2}}, \quad z \in \mathbb{C} \backslash \mathbb{R}
$$

Clearly, we may just as well write the function $m_{ \pm}$as the limit

$$
m_{ \pm}(z)= \pm \lim _{x \rightarrow \mp \infty} \frac{\theta_{ \pm}^{[ \pm]}(z, x)}{z \phi_{ \pm}^{[ \pm]}(z, x)}=\mp \lim _{\xi \rightarrow \mp \infty} \frac{Y_{ \pm, 21}(z, \xi)}{Y_{ \pm, 22}(z, \xi)}, \quad z \in \mathbb{C} \backslash \mathbb{R} .
$$

Now we observe that (2.24) gives for every $\xi \in \mathbb{R}_{\mp}$ the expansion

$$
\frac{Y_{ \pm, 21}(z, \xi)}{Y_{ \pm, 22}(z, \xi)}=z \int_{0}^{\xi} \mathrm{a}_{ \pm}(s)^{2} \mathrm{~d} s+z \int_{0}^{\xi} \mathrm{d} \beta_{ \pm}+\mathcal{O}\left(z^{2}\right), \quad z \rightarrow 0
$$

Since the convergence in (2.28) is uniform for all $z \in \mathbb{C} \backslash \mathbb{R}$ that lie in a small neighborhood of zero, we infer, after a substitution, that $m_{ \pm}$has the expansion

$$
m_{ \pm}(z)=z \int_{\mathbb{R}} \mathrm{e}^{ \pm x}\left(u^{\prime}(x) \mp u(x)\right)^{2} \mathrm{~d} x+z \int_{\mathbb{R}} \mathrm{e}^{ \pm x} \mathrm{~d} v(x)+\mathcal{O}\left(z^{2}\right), \quad z \rightarrow 0 .
$$

Upon differentiating (2.27) and letting $z \rightarrow 0$, we obtain the identity in (2.25).

In this context, let us also mention the following result which characterizes the subclass of $\mathcal{E}$ that gives rise to purely positive/negative spectrum.

Proposition 2.7. The spectrum $\sigma$ is positive/negative if and only if the Borel measure $v$ vanishes identically and the distribution $\omega$ is non-negative/non-positive. In this case, the distribution $\omega$ can be represented by a non-negative/non-positive finite Borel measure on $\mathbb{R}$ (for simplicity denoted with $\omega$ as well) and

$$
\sum_{\lambda \in \sigma} \frac{1}{\lambda}=\int_{\mathbb{R}} \mathrm{d} \omega .
$$


Proof. If $v$ vanishes identically and $\omega$ is non-negative/non-positive, then (2.16) and (2.17) show that the spectrum is positive/negative. Conversely, if the spectrum is positive/negative, then [35, Lemma 7.2] shows that the measures $\beta_{+}$and $\beta_{-}$ vanish identically as well as that the functions $a_{+}$and $a_{-}$have non-decreasing/nonincreasing representatives. Clearly, the measures $\beta_{+}$and $\beta_{-}$vanish identically if and only if so does $v$. Given some $h \in H^{1}(\mathbb{R})$ with compact support, we set

$$
h_{ \pm}(\xi)=\mp \xi h\left(\eta_{ \pm}(\xi)\right), \quad \xi \in \mathbb{R}_{\mp},
$$

and note that

$$
\omega(h)=-\frac{1}{2} \int_{\mathbb{R}_{-}} h_{+}^{\prime}(\xi) \mathrm{a}_{+}(\xi) \mathrm{d} \xi-\frac{1}{2} \int_{\mathbb{R}_{+}} h_{-}^{\prime}(\xi) \mathrm{a}_{-}(\xi) \mathrm{d} \xi .
$$

This shows that $\omega$ is a non-negative/non-positive distribution and therefore can be represented by a non-negative/non-positive Borel measure on $\mathbb{R}$. Now for every $k \in \mathbb{N}$ let $h_{k} \in H^{1}(\mathbb{R})$ be the piecewise linear function such that $h_{k}$ is equal to one on $[-k, k]$, equal to zero outside of $[-k-1, k+1]$ and linear in between. Then from

$$
\int_{\mathbb{R}} h_{k} \mathrm{~d} \omega=\int_{\mathbb{R}} u(x) h_{k}(x) \mathrm{d} x+\int_{\mathbb{R}} u^{\prime}(x) h_{k}^{\prime}(x) \mathrm{d} x
$$

we see that $\omega$ is a finite measure as well as (2.29) upon letting $k \rightarrow \infty$.

An identity similar to (2.29) also holds under the sole assumption that the distribution $\omega$ can be represented by a real-valued Borel measure on $\mathbb{R}$ (again denoted with $\omega$ as well) with finite total variation. More precisely, one has the equality

$$
\sum_{\lambda \in \sigma}^{\leftrightarrow} \frac{1}{\lambda}=\int_{\mathbb{R}} \mathrm{d} \omega
$$

in this case, which follows readily from the first trace formula in Proposition 2.5.

\section{The Inverse Spectral Problem}

We are now going to solve the corresponding inverse spectral problem for the class $\mathcal{E}$. Let us point out that the given sufficient conditions on the spectral data are also necessary in view of Corollary 2.2 (in conjunction with well-known properties of entire functions of Cartwright class; for example, see [12, Chapter 8] or [60, Theorem 17.2.1]) as well as Proposition 2.6 and Lemma 2.4. Thus, we indeed obtain a complete characterization of all possible spectral data for the class $\mathcal{E}$.

Theorem 3.1. Let $\sigma$ be a discrete set of nonzero reals such that the limit ${ }^{2}$

$$
\lim _{r \rightarrow \infty} \frac{n_{\sigma}(r)}{r}
$$

\footnotetext{
${ }^{2}$ Recall that $n_{\sigma}(r)$ denotes the number of all $\lambda \in \sigma$ with modulus not greater than $r$.
} 
exists in $[0, \infty)$ and such that the entire function $W$ is well-defined by ${ }^{3}$

$$
W(z)=\prod_{\lambda \in \sigma}^{\leftrightarrow}\left(1-\frac{z}{\lambda}\right), \quad z \in \mathbb{C} .
$$

Moreover, for each $\lambda \in \sigma$ let $\kappa_{\lambda} \in \mathbb{R}$ such that the sum

$$
\sum_{\lambda \in \sigma} \frac{1}{\lambda^{2}} \frac{\mathrm{e}^{\left|\kappa_{\lambda}\right|}}{|\lambda \dot{W}(\lambda)|}
$$

is finite. Then there is a unique pair $(u, \mu) \in \mathcal{E}$ such that the associated spectrum coincides with $\sigma$ and the logarithmic coupling constants are $\kappa_{\lambda}$ for each $\lambda \in \sigma$.

Proof. Uniqueness. Since the given spectral data uniquely determines the WeylTitchmarsh function $m_{-}$in view of (2.27) and Lemma 2.4, the uniqueness part in [35, Theorem 6.1] shows that the function $a_{-}$and the Borel measure $\beta_{-}$are uniquely determined as well. It follows readily from the definition of $\beta_{-}$in (2.4) that this also uniquely determines the Borel measure $v$. Furthermore, because of

$$
u\left(\eta_{ \pm}(\xi)\right)=-\frac{1}{\xi} \int_{0}^{\xi} \mathrm{a}_{ \pm}(s) s \mathrm{~d} s, \quad \xi \in \mathbb{R}_{\mp},
$$

we conclude that the function $u$ is uniquely determined too and thus so is $\mu$. Existence. Let us introduce the meromorphic Herglotz-Nevanlinna function

$$
m(z)=\sum_{\lambda \in \sigma} \frac{z}{\lambda(\lambda-z)} \frac{\mathrm{e}^{-\kappa_{\lambda}}}{|\lambda \dot{W}(\lambda)|}, \quad z \in \mathbb{C} \backslash \mathbb{R} .
$$

From the existence part of [35, Theorem 6.1], we obtain a real-valued and locally square integrable function a on $[0, \infty)$ and a non-negative Borel measure $\beta$ on $[0, \infty)$ with $\beta(\{0\})=0$ such that the function $m$ is the Weyl-Titchmarsh function for

$$
\begin{aligned}
Y(z, \xi)= & \left(\begin{array}{ll}
1 & 0 \\
0 & 1
\end{array}\right)+z \int_{0}^{\xi}\left(\begin{array}{cc}
-\mathrm{a}(s) & -1 \\
\mathrm{a}(s)^{2} & \mathrm{a}(s)
\end{array}\right) Y(z, s) \mathrm{d} s \\
& +z \int_{0}^{\xi}\left(\begin{array}{ll}
0 & 0 \\
1 & 0
\end{array}\right) Y(z, s) \mathrm{d} \beta(s), \quad \xi \in[0, \infty),
\end{aligned}
$$

that is, if $Y(z, \cdot)$ denotes the unique solution of the integral equation (3.5) for each $z \in \mathbb{C}$, then the function $m$ is given by

$$
m(z)=\lim _{\xi \rightarrow \infty} \frac{Y_{11}(z, \xi)}{Y_{12}(z, \xi)}, \quad z \in \mathbb{C} \backslash \mathbb{R} .
$$

In order to state a required fact from [35] in a concise way, we introduce the set $\Sigma \subseteq(0, \infty)$ that consists of all $\xi \in(0, \infty)$ such that the function a is not equal

\footnotetext{
3 If the limit in (3.1) exists in [0, ), then this is the case if and only if the sum $\sum_{\lambda \in \sigma}^{\leftrightarrow} \frac{1}{\lambda}$ exists.
} 
to a constant almost everywhere near $\xi$ or such that $\xi$ belongs to the topological support of $\beta$. Let $f_{1}, f_{2} \in L^{2}(0, \infty)$ be orthogonal to all $h \in L^{2}(0, \infty)$ that satisfy

$$
\int_{0}^{\xi} h(s) \mathrm{d} s=0, \quad \xi \in \Sigma,
$$

and functions $g_{1}, g_{2}$ on $[0, \infty)$ be square integrable with respect to $\beta$. Upon setting

$$
F_{i}(\lambda)=\int_{0}^{\infty} \frac{Y_{12}^{\prime}(\lambda, \xi)}{\lambda} f_{i}(\xi) \mathrm{d} \xi+\int_{0}^{\infty} Y_{12}(\lambda, \xi) g_{i}(\xi) \mathrm{d} \beta(\xi), \quad \lambda \in \sigma, i=1,2,
$$

we have the identity

$$
\sum_{\lambda \in \sigma} F_{1}(\lambda) F_{2}(\lambda)^{*} \frac{\mathrm{e}^{-\kappa \lambda}}{|\lambda \dot{W}(\lambda)|}=\int_{0}^{\infty} f_{1}(\xi) f_{2}(\xi)^{*} \mathrm{~d} \xi+\int_{0}^{\infty} g_{1}(\xi) g_{2}(\xi)^{*} \mathrm{~d} \beta(\xi)
$$

since the spectral transform introduced in [35, Section 5] is a partial isometry.

Motivated by the relation (3.4), we define the real-valued function $u$ on $\mathbb{R}$ via

$$
u(\ln (\xi))=-\frac{1}{\xi} \int_{0}^{\xi} \mathrm{a}(s) s \mathrm{~d} s, \quad \xi \in(0, \infty),
$$

so that $u$ belongs to $H_{\text {loc }}^{1}(\mathbb{R})$ and satisfies

$$
\mathrm{a}(\xi)=\frac{-u^{\prime}(\ln (\xi))-u(\ln (\xi))}{\xi}
$$

for almost all $\xi \in(0, \infty)$. Furthermore, we define the Borel measure $\mu$ on $\mathbb{R}$ by (1.7), where $v$ is the Borel measure on $\mathbb{R}$ such that that we have

$$
\beta(B)=\int_{\ln (B)} \mathrm{e}^{-x} \mathrm{~d} v(x)
$$

for every Borel set $B \subseteq(0, \infty)$. Since the function a is square integrable near zero and the Borel measure $\beta$ is finite near zero, it follows readily that

$$
\int_{-\infty}^{c} \mathrm{e}^{-x}\left(u^{\prime}(x)+u(x)\right)^{2} \mathrm{~d} x+\int_{-\infty}^{c} \mathrm{e}^{-x} \mathrm{~d} v(x)<\infty
$$

for every $c \in \mathbb{R}$. In particular, this guarantees that the function $u$ lies in $H^{1}(\mathbb{R})$ near $-\infty$ (more precisely, one sees from (3.8) that $u$ decays exponentially near $-\infty$ and since $u^{\prime}+u$ is clearly square integrable near $-\infty$, we conclude that $u^{\prime}$ is as well) and that the measure $\mu$ is finite near $-\infty$. We are now left to verify that the pair $(u, \mu)$ actually belongs to $\mathcal{E}$. In fact, in this case it is readily seen that a coincides with the function $\mathrm{a}_{-}$as introduced by (2.3) and that $\beta$ coincides with the Borel measure $\beta_{-}$as introduced by (2.4). From (2.26), we then see that the function $m$ coincides with the Weyl-Titchmarsh function $m_{-}$for this pair as introduced in the proof of Proposition 2.6. Upon taking (2.27) and Lemma 2.4 into account, one then may conclude that the pair $(u, \mu)$ indeed gives rise to the desired spectral data. 
In order to show that the pair $(u, \mu)$ belongs to the set $\mathcal{E}$, let us first consider the special case when $a$ is equal to some constant $a_{0} \in \mathbb{R}$ almost everywhere near $\infty$ and $\beta$ vanishes near $\infty$. From this we readily infer that

$$
Y(z, \xi)=\left(\begin{array}{cc}
1-z \mathrm{a}_{0}\left(\xi-\xi_{0}\right) & -z\left(\xi-\xi_{0}\right) \\
z \mathrm{a}_{0}^{2}\left(\xi-\xi_{0}\right) & 1+z \mathrm{a}_{0}\left(\xi-\xi_{0}\right)
\end{array}\right) Y\left(z, \xi_{0}\right)
$$

as long as $\xi$ and $\xi_{0}$ are close enough to $\infty$. Thus, the function $m$ is given by

$$
m(z)=\frac{\mathrm{a}_{0} Y_{11}\left(z, \xi_{0}\right)+Y_{21}\left(z, \xi_{0}\right)}{\mathrm{a}_{0} Y_{21}\left(z, \xi_{0}\right)+Y_{22}\left(z, \xi_{0}\right)}, \quad z \in \mathbb{C} \backslash \mathbb{R},
$$

and evaluating the limit as $z \rightarrow 0$ in this equation shows that $\mathrm{a}_{0}$ has to be zero, which immediately implies that the pair $(u, \mu)$ belongs to $\mathcal{E}$. In particular, this proves the claim in the case when the set $\sigma$ is finite (since then the set $\Sigma$ is finite as well). Thus, back in the general case, for every $k \in \mathbb{N}$ we may find a $\left(u_{k}, \mu_{k}\right) \in \mathcal{E}$ such that the corresponding Weyl-Titchmarsh function $m_{k,-}$ is given by

$$
m_{k,-}(z)=\sum_{\substack{\lambda \in \sigma \\|\lambda| \leqq k}} \frac{z}{\lambda(\lambda-z)} \frac{\mathrm{e}^{-\kappa_{\lambda}}}{|\lambda \dot{W}(\lambda)|}, \quad z \in \mathbb{C} \backslash \mathbb{R}
$$

With the functions $a_{k,-}$ defined as in (2.3), we infer from [35, Proposition 6.2] that

$$
\lim _{k \rightarrow \infty} \int_{0}^{\xi} \mathrm{a}_{k,-}(s) \mathrm{d} s=\int_{0}^{\xi} \mathrm{a}(s) \mathrm{d} s
$$

locally uniformly for all $\xi \in[0, \infty)$. In view of (3.4) and (3.8), this shows that the functions $u_{k}$ converge pointwise to $u$ and since they are uniformly bounded in $H^{1}(\mathbb{R})$ by Proposition 2.5, a compactness argument shows that $u$ lies in $H^{1}(\mathbb{R})$.

To verify also the remaining growth restrictions on $(u, \mu)$ in the general case, we now may assume that there is an increasing sequence $x_{n} \rightarrow \infty$ such that $\xi_{n}=\mathrm{e}^{x_{n}}$ belongs to $\Sigma$ for every $n \in \mathbb{N}_{0}$. We fix some $n \in \mathbb{N}_{0}$ and define the function $J_{n}$ by

$$
J_{n}(\zeta, z)=\frac{Z_{1}\left(z, \xi_{n}\right) Z_{2}\left(\zeta, \xi_{n}\right)^{*}-Z_{2}\left(z, \xi_{n}\right) Z_{1}\left(\zeta, \xi_{n}\right)^{*}}{z-\zeta^{*}}, \quad \zeta, z \in \mathbb{C} \backslash \mathbb{R}
$$

where $Z(z, \cdot)$ is the Weyl solution given by

$$
Z(z, \xi)=Y(z, \xi)\left(\begin{array}{c}
z W(z) \\
-V(z)
\end{array}\right), \quad \xi \in[0, \infty),
$$

for every $z \in \mathbb{C}$, and $V$ is the entire function defined in such a way that

$$
V(z)=z W(z) m(z), \quad z \in \mathbb{C} \backslash \mathbb{R} .
$$

Because the function $Z(z, \cdot)$ is a Weyl solution for every $z \in \mathbb{C} \backslash \mathbb{R}$, we note that $J_{n}$ can be rewritten as (see the proof of [35, Lemma 5.1])

$$
J_{n}(\zeta, z)=\int_{\xi_{n}}^{\infty} \frac{Z_{1}^{\prime}(z, s)}{z} \frac{Z_{1}^{\prime}(\zeta, s)^{*}}{\zeta^{*}} \mathrm{~d} s+\int_{\xi_{n}}^{\infty} Z_{1}(z, s) Z_{1}(\zeta, s)^{*} \mathrm{~d} \beta(s)
$$


for all $\zeta, z \in \mathbb{C} \backslash \mathbb{R}$. In particular, this implies that the entire function $E_{n}$ defined by

$$
E_{n}(z)=z \psi\left(z, x_{n}\right)-\mathrm{i} \psi^{[+]}\left(z, x_{n}\right), \quad z \in \mathbb{C},
$$

is a de Branges function, where $\psi(z, \cdot)$ is the solution of the differential equation (2.1) so that (compare the proof of Theorem 2.1)

$$
\left(\begin{array}{c}
z \psi(z, x) \\
-\psi^{[-]}(z, x)
\end{array}\right)=\left(\begin{array}{cc}
\mathrm{e}^{-\frac{x}{2}} & 0 \\
0 & \mathrm{e}^{\frac{x}{2}}
\end{array}\right) Z\left(z, \mathrm{e}^{x}\right), \quad x \in \mathbb{R},
$$

for each $z \in \mathbb{C}$, with the quantities $\omega$ and $v$ defined as in (A.1) and (1.7). The reproducing kernel $K_{n}$ in the corresponding de Branges space $\mathcal{B}_{n}$ is given by

$$
K_{n}(\zeta, z)=J_{n}(\zeta, z)+\psi\left(z, x_{n}\right) \psi\left(\zeta, x_{n}\right)^{*}, \quad \zeta, z \in \mathbb{C} \backslash \mathbb{R} .
$$

An integration by parts and the integral equation (3.5) show that for every $z \in$ $\mathbb{C} \backslash \mathbb{R}$ the function $Z_{1}^{\prime}(z, \cdot)$ restricted to $\left(\xi_{n}, \infty\right)$ is orthogonal to all functions $h \in$ $L^{2}(0, \infty)$ that satisfy (3.6). Thus, the identity (3.7) for the particular functions

$$
f_{i}(s)=\frac{1}{z_{i}}\left\{\begin{array}{ll}
Z_{1}\left(z_{i}, \xi_{n}\right) \mathrm{e}^{-x_{n}}, & s \in\left[0, \xi_{n}\right), \\
Z_{1}^{\prime}\left(z_{i}, s\right), & s \in\left[\xi_{n}, \infty\right),
\end{array} \quad g_{i}(s)= \begin{cases}0, & s \in\left[0, \xi_{n}\right), \\
Z_{1}\left(z_{1}, s\right), & s \in\left[\xi_{n}, \infty\right),\end{cases}\right.
$$

with $z_{1}=z^{*}$ and $z_{2}=\zeta^{*}$ gives (note that $Z_{1}(\lambda, \cdot)=-V(\lambda) Y_{12}(\lambda, \cdot$ ) for all $\lambda \in \sigma)$

$$
\begin{aligned}
\sum_{\lambda \in \sigma} \frac{K_{n}(z, \lambda)}{V(\lambda)} \frac{K_{n}(\zeta, \lambda)^{*}}{V(\lambda)^{*}} \frac{\mathrm{e}^{-\kappa_{\lambda}}}{|\lambda \dot{W}(\lambda)|} & =K_{n}(z, \zeta) \\
& =\left\langle K_{n}(z, \cdot), K_{n}(\zeta, \cdot)\right\rangle_{\mathcal{B}_{n}}, \quad \zeta, z \in \mathbb{C} \backslash \mathbb{R} .
\end{aligned}
$$

The values of $V$ on the set $\sigma$ are readily evaluated and we obtain

$$
\sum_{\lambda \in \sigma}|F(\lambda)|^{2} \frac{\mathrm{e}^{\kappa_{\lambda}}}{|\lambda \dot{W}(\lambda)|}=\|F\|_{\mathcal{B}_{n}}^{2}, \quad F \in \mathcal{B}_{n}
$$

after employing simple linearity, continuity and density arguments.

Now let $H$ be a locally integrable, trace normed, real, symmetric and nonnegative definite $2 \times 2$ matrix function on $[0, \infty)$ such that if $M(z, \cdot)$ denotes the unique solution of the integral equation

$$
M(z, t)=\left(\begin{array}{ll}
1 & 0 \\
0 & 1
\end{array}\right)+z \int_{0}^{t}\left(\begin{array}{cc}
0 & -1 \\
1 & 0
\end{array}\right) H(s) M(z, s) \mathrm{d} s, \quad t \in[0, \infty),
$$

for every $z \in \mathbb{C}$, then we have

$$
\lim _{t \rightarrow \infty} \frac{M_{11}(z, t)}{M_{12}(z, t)}=\sum_{\lambda \in \sigma} \frac{z}{\lambda(\lambda-z)} \frac{\mathrm{e}^{\kappa_{\lambda}}}{|\lambda \dot{W}(\lambda)|}, \quad z \in \mathbb{C} \backslash \mathbb{R} .
$$

Such a function $H$ is guaranteed to exist by the solution of the inverse problem for canonical systems due to de Branges; see [30, Theorem XII], [69, Theorem 2.4]. Finiteness of the sum in (3.3) implies (see [56, Lemma 5.5], [66, Theorem 6.17] and 
note that existence of (3.1) and (3.2) together establish [56, Condition (C2)]; cf. $[12, \S 8.3 .6])$ that the entire function $W$ belongs to the Cartwright class. Moreover, since $m$ is a Herglotz-Nevanlinna function, so does the function $V$ and thus also $E_{n}$ for every $n \in \mathbb{N}_{0}$. In view of [31, Theorem VII] in conjunction with (3.9) and upon employing [29, Theorem I], we obtain a $t_{n} \in(0, \infty)$ so that

$$
\left(\begin{array}{c}
z \psi\left(z, x_{n}\right) \\
-\psi^{[+]}\left(z, x_{n}\right)
\end{array}\right)=\Gamma_{n}\left(\begin{array}{l}
M_{12}\left(z, t_{n}\right) \\
M_{22}\left(z, t_{n}\right)
\end{array}\right), \quad z \in \mathbb{C},
$$

for a real $2 \times 2$ matrix $\Gamma_{n}$ with $\operatorname{det} \Gamma_{n}=-1$. Evaluating at zero shows that

$$
\Gamma_{n}=\left(\begin{array}{cc}
-\mathrm{e}^{\frac{x_{n}}{2}} & 0 \\
\varepsilon_{n} & \mathrm{e}^{-\frac{x_{n}}{2}}
\end{array}\right)
$$

for some $\varepsilon_{n} \in \mathbb{R}$. We note that the sequence $t_{n}$ may be chosen in such a way that it is non-increasing because the sequence of de Branges spaces $\mathcal{B}_{n}$ is non-increasing with respect to inclusion. Thus, for every $n \in \mathbb{N}_{0}$ we have on the one side

$$
\left(\begin{array}{c}
z \psi\left(z, x_{0}\right) \\
-\psi^{[+]}\left(z, x_{0}\right)
\end{array}\right)=\Gamma_{0} M\left(z, t_{0}\right) M\left(z, t_{n}\right)^{-1} \Gamma_{n}^{-1}\left(\begin{array}{c}
z \psi\left(z, x_{n}\right) \\
-\psi^{[+]}\left(z, x_{n}\right)
\end{array}\right), \quad z \in \mathbb{C},
$$

and on the other side (due to Lemma A.2) also

$$
\left(\begin{array}{c}
z \psi\left(z, x_{0}\right) \\
-\psi^{[+]}\left(z, x_{0}\right)
\end{array}\right)=\Omega_{+}\left(z, x_{0}, x_{n}\right)\left(\begin{array}{c}
z \psi\left(z, x_{n}\right) \\
-\psi^{[+]}\left(z, x_{n}\right)
\end{array}\right), \quad z \in \mathbb{C},
$$

where $\Omega_{ \pm}\left(z, \cdot, x_{n}\right)$ denotes the matrix valued solution of the system (A.4) such that $\Omega_{ \pm}\left(z, x_{n}, x_{n}\right)$ is the identity matrix. In view of [28, Problem 100], this gives

$$
M\left(z, t_{0}\right) M\left(z, t_{n}\right)^{-1}=\Gamma_{0}^{-1} \Omega_{+}\left(z, x_{0}, x_{n}\right) \Gamma_{n}, \quad z \in \mathbb{C} .
$$

Differentiating with respect to $z$ and evaluating at zero, we obtain

$$
\begin{aligned}
\int_{t_{n}}^{t_{0}} H(s) \mathrm{d} s= & \int_{x_{0}}^{x_{n}} \mathrm{e}^{-s} \mathrm{~d} s\left(\begin{array}{cc}
\varepsilon_{0} \mathrm{e}^{\frac{x_{0}}{2}} \varepsilon_{n} \mathrm{e}^{\frac{x_{n}}{2}} & \varepsilon_{0} \mathrm{e}^{\frac{x_{0}}{2}} \\
\varepsilon_{n} \mathrm{e}^{\frac{x_{n}}{2}} & 1
\end{array}\right) \\
& +\int_{x_{0}}^{x_{n}} u^{\prime}(s)-u(s) \mathrm{d} s\left(\begin{array}{cc}
\varepsilon_{0} \mathrm{e}^{\frac{x_{0}}{2}}+\varepsilon_{n} \mathrm{e}^{\frac{x_{n}}{2}} & 1 \\
1 & 0
\end{array}\right) \\
& +\int_{x_{0}}^{x_{n}} \mathrm{e}^{s}\left(u^{\prime}(s)-u(s)\right)^{2} \mathrm{~d} s\left(\begin{array}{ll}
1 & 0 \\
0 & 0
\end{array}\right)+\int_{x_{0}}^{x_{n}} \mathrm{e}^{s} \mathrm{~d} v(s)\left(\begin{array}{ll}
1 & 0 \\
0 & 0
\end{array}\right) .
\end{aligned}
$$

Since $H$ is integrable near zero, the top-right entry shows that the integrals

$$
\int_{x_{0}}^{x_{n}} u^{\prime}(s)-u(s) \mathrm{d} s
$$

are uniformly bounded for all $n \in \mathbb{N}_{0}$. Moreover, from the bottom-left entry we now infer that the quantities $\varepsilon_{n} \mathrm{e}^{\frac{x_{n}}{2}}$ are uniformly bounded for all $n \in \mathbb{N}_{0}$ as well. Finally, the top-left entry implies that the sum

$$
\int_{x_{0}}^{x_{n}} \mathrm{e}^{s}\left(u^{\prime}(s)-u(s)\right)^{2} \mathrm{~d} s+\int_{x_{0}}^{x_{n}} \mathrm{e}^{s} \mathrm{~d} v(s)
$$


is uniformly bounded for all $n \in \mathbb{N}_{0}$, which concludes the proof since $x_{n} \rightarrow \infty$.

For a discrete set $\sigma$ of nonzero reals such that the limit (3.1) exists in $[0, \infty)$ and such that the entire function $W$ is well-defined by (3.2), we define the isospectral set Iso $(\sigma)$ as the set of all those pairs $(u, \mu) \in \mathcal{E}$ whose associated spectra coincide with the set $\sigma$. It is an immediate consequence of Theorem 3.1 that the isospectral set $\operatorname{Iso}(\sigma)$ is in one-to-one correspondence with the set ${ }^{4}$

$$
\Lambda_{\sigma}=\left\{\kappa \in \mathbb{R}^{\sigma} \mid \sum_{\lambda \in \sigma} \frac{1}{\lambda^{2}} \frac{\mathrm{e}^{\left|\kappa_{\lambda}\right|}}{|\lambda \dot{W}(\lambda)|}<\infty\right\},
$$

by means of the bijection given by

$$
(u, \mu) \mapsto\left\{\kappa_{\lambda}\right\}_{\lambda \in \sigma}
$$

Of course, the solution of the inverse spectral problem can easily be formulated in terms of the right/left norming constants as well. However, the picture (that is, the condition corresponding to (3.3) on the asymptotic behavior) looks less symmetric.

Corollary 3.2. Let $\sigma$ be a discrete set of nonzero reals such that the limit (3.1) exists in $[0, \infty)$ and such that the entire function $W$ is well-defined by (3.2). Moreover, for each $\lambda \in \sigma$ let $\gamma_{\lambda, \pm}^{2} \in \mathbb{R}$ such that $\lambda \gamma_{\lambda, \pm}^{2}>0$ and the sums

$$
\sum_{\lambda \in \sigma} \frac{1}{\lambda^{2}} \frac{1}{\lambda \gamma_{\lambda, \pm}^{2}}, \quad \quad \sum_{\lambda \in \sigma} \frac{1}{\lambda^{2}} \frac{\gamma_{\lambda, \pm}^{2}}{\lambda \dot{W}(\lambda)^{2}}
$$

are finite. Then there is a unique pair $(u, \mu) \in \mathcal{E}$ such that the associated spectrum coincides with $\sigma$ and the right/left norming constants are $\gamma_{\lambda, \pm}^{2}$ for each $\lambda \in \sigma$.

In order to avoid misunderstandings, let us point out that this corollary has to be read as two separate statements according to the plus-minus alternative, that is, we are only able to prescribe either the right or the left norming constants.

Remark 3.3. If the set $\sigma$ is finite, then all conditions in Theorem 3.1 and Corollary 3.2 are trivially satisfied. The solution of the inverse spectral problem in this case has a particular simple form and can be written down explicitly in terms of the spectral data; see [33, Section 4]. We will see next that these kinds of solutions can be used to approximate solutions in the general case in a certain way.

We conclude this section by establishing a continuity property for the inverse spectral transform. In order to state it, let $\left(u_{k}, \mu_{k}\right)$ belong to $\mathcal{E}$ for every $k \in \mathbb{N}$ and denote all corresponding quantities in an obvious way with an additional subscript.

4 To be precise, this has to be interpreted appropriately in the trivial case when $\sigma$ is empty. 
Proposition 3.4. We have

$$
\begin{gathered}
\sum_{\lambda \in \sigma_{k}} \frac{1}{\lambda\left(1+\lambda^{2}\right)} \frac{1}{\lambda \gamma_{k, \lambda, \pm}^{2}} \rightarrow \sum_{\lambda \in \sigma} \frac{1}{\lambda\left(1+\lambda^{2}\right)} \frac{1}{\lambda \gamma_{\lambda, \pm}^{2}}, \\
\sum_{\lambda \in \sigma_{k}} \frac{\chi(\lambda)}{1+\lambda^{2}} \frac{1}{\lambda \gamma_{k, \lambda, \pm}^{2}} \rightarrow \sum_{\lambda \in \sigma} \frac{\chi(\lambda)}{1+\lambda^{2}} \frac{1}{\lambda \gamma_{\lambda, \pm}^{2}},
\end{gathered}
$$

as $k \rightarrow \infty$ for all functions $\chi \in C(\mathbb{R})$ such that the limit of $\chi(\lambda)$ as $|\lambda| \rightarrow \infty$ exists and is finite if and only if the functions $u_{k}$ converge to $u$ pointwise and

$$
\begin{aligned}
\int_{ \pm \infty}^{x} \mathrm{e}^{\mp s} & \left(\int_{ \pm \infty}^{s} \mathrm{e}^{ \pm r}\left(u_{k}^{\prime}(r) \mp u_{k}(r)\right)^{2} \mathrm{~d} r+\int_{ \pm \infty}^{s} \mathrm{e}^{ \pm r} d v_{k}(r)\right) \mathrm{d} s \\
& \rightarrow \int_{ \pm \infty}^{x} \mathrm{e}^{\mp s}\left(\int_{ \pm \infty}^{s} \mathrm{e}^{ \pm r}\left(u^{\prime}(r) \mp u(r)\right)^{2} \mathrm{~d} r+\int_{ \pm \infty}^{s} \mathrm{e}^{ \pm r} d v(r)\right) \mathrm{d} s
\end{aligned}
$$

as $k \rightarrow \infty$ for all $x \in \mathbb{R}$. In this case, the functions $u_{k}$ converge to $u$ even locally uniformly and the Borel measures $\mu_{k}$ converge to $\mu$ in the sense of distributions.

Proof. It follows from [35, Proposition 6.2] that the first condition on convergence of (3.13) and (3.14) is equivalent to

$$
\begin{aligned}
& \int_{0}^{\xi} \mathrm{a}_{k, \pm}(s) \mathrm{d} s \rightarrow \int_{0}^{\xi} \mathrm{a}_{ \pm}(s) \mathrm{d} s \\
& \int_{0}^{\xi}\left(\int_{0}^{s} \mathrm{a}_{k, \pm}(r)^{2} \mathrm{~d} r+\int_{0}^{s} \mathrm{~d} \beta_{k, \pm}\right) \mathrm{d} s \rightarrow \int_{0}^{\xi}\left(\int_{0}^{s} \mathrm{a}_{ \pm}(r)^{2} \mathrm{~d} r+\int_{0}^{s} \mathrm{~d} \beta_{ \pm}\right) \mathrm{d} s,
\end{aligned}
$$

as $k \rightarrow \infty$ for all $\xi \in \mathbb{R}_{\mp}$, in which case the convergence is uniform as long as $\xi$ stays bounded. In conjunction with (3.4), one sees that this implies that the sequence $u_{k}(x)$ converges to $u(x)$ for all $x \in \mathbb{R}$. Moreover, upon employing a substitution in (3.17), we readily infer that (3.15) holds as $k \rightarrow \infty$ for all $x \in \mathbb{R}$ as well. Both of these convergences are uniform as long as $x$ stays away from $\mp \infty$. In addition, for every smooth function $h$ on $\mathbb{R}$ that vanishes near $\mp \infty$ and such that $h^{\prime}$ has compact support we have the identity

$$
\begin{aligned}
\int_{\mathbb{R}} h \mathrm{~d} \mu_{k} & =\int_{\mathbb{R}} h(x) \alpha_{k, \pm}(x)^{2} \mathrm{~d} x \pm 2 \int_{\mathbb{R}} h(x) u_{k}(x) u_{k}^{\prime}(x) \mathrm{d} x+\int_{\mathbb{R}} h \mathrm{~d} v_{k} \\
& =\int_{\mathbb{R}_{\mp}} h_{ \pm}(\xi) \mathrm{a}_{k, \pm}(\xi)^{2} \mathrm{~d} \xi \mp \int_{\mathbb{R}} h^{\prime}(x) u_{k}(x)^{2} \mathrm{~d} x+\int_{\mathbb{R}_{\mp}} h_{ \pm} \mathrm{d} \beta_{k, \pm} \\
& =\int_{\mathbb{R}_{\mp}} h_{ \pm}^{\prime \prime}(\xi) \int_{0}^{\xi}\left(\int_{0}^{s} \mathrm{a}_{k, \pm}(r)^{2} \mathrm{~d} r+\int_{0}^{s} \mathrm{~d} \beta_{k, \pm}\right) \mathrm{d} s \mathrm{~d} \xi \\
& \mp \int_{\mathbb{R}} h^{\prime}(x) u_{k}(x)^{2} \mathrm{~d} x
\end{aligned}
$$

${ }^{5}$ Let us mention that the following condition is equivalent to locally uniform convergence $m_{k, \pm} \rightarrow m_{ \pm}$of the corresponding Weyl-Titchmarsh functions given as in (2.27). 
with $h_{ \pm}$given by (2.30). Taking the limit $k \rightarrow \infty$ on the right-hand side gives

$$
\int_{\mathbb{R}} h \mathrm{~d} \mu_{k} \rightarrow \int_{\mathbb{R}} h \mathrm{~d} \mu, \quad k \rightarrow \infty,
$$

which shows that the Borel measures $\mu_{k}$ converge to $\mu$ in the sense of distributions.

For the converse direction, we now assume that the functions $u_{k}$ converge to $u$ pointwise and that (3.15) holds as $k \rightarrow \infty$ for every $x \in \mathbb{R}$. As observed above, the latter condition is equivalent to the fact that (3.17) holds as $k \rightarrow \infty$ for all $\xi \in \mathbb{R}_{\mp}$. Upon expressing the integrals in (3.16) in terms of $u_{k}$ and $u$ by using (2.3) as well as a substitution, we infer (use Lebesgue's dominated convergence theorem and note that convergence of (3.17) implies a uniform bound on the monotone integrands which yields a sufficient estimate for the functions $u_{k}$ ) that (3.16) holds as $k \rightarrow \infty$ for all $\xi \in \mathbb{R}_{\mp}$, which establishes the equivalence in the claim.

We endow $\mathcal{E}$ with the unique first countable topology ${ }^{6}$ such that the sequence $\left(u_{k}, \mu_{k}\right)$ converges to $(u, \mu)$ if and only if the functions $u_{k}$ converge to $u$ pointwise and both of the plus-minus alternatives in (3.15) hold as $k \rightarrow \infty$ for all $x \in \mathbb{R}$.

Corollary 3.5. If the sequence $\left(u_{k}, \mu_{k}\right)$ converges to $(u, \mu)$ in $\mathcal{E}$, then the functions $u_{k}$ converge to $u$ uniformly as well as weakly in $H^{1}(\mathbb{R})$, the Borel measures $\mu_{k}$ converge to $\mu$ in the weak $k^{*}$ topology $y^{7}$ and the sequence $\mu_{k}(\mathbb{R})$ converges to $\mu(\mathbb{R})$.

Proof. We have seen in the first part of the proof of Proposition 3.4 that the given assumption implies that the functions $u_{k}$ converge to $u$ uniformly. After choosing a suitable partition of unity (two functions are sufficient) we infer from (3.18) that the sequence $\mu_{k}(\mathbb{R})$ converges to $\mu(\mathbb{R})$. In particular, the sequence $\mu_{k}(\mathbb{R})$ is bounded which allows to deduce the remaining claims in a straightforward manner.

\section{The Conservative Camassa-Holm Flow}

Let us define the conservative Camassa-Holm flow $\Phi$ on $\mathcal{E}$ as a mapping

$$
\Phi: \mathcal{E} \times \mathbb{R} \rightarrow \mathcal{E}
$$

in the following way: given a pair $(u, \mu) \in \mathcal{E}$ with associated spectrum $\sigma$ and logarithmic coupling constants $\kappa_{\lambda}$ for every $\lambda \in \sigma$, as well as a $t \in \mathbb{R}$, the corresponding image $\Phi^{t}(u, \mu)$ under $\Phi$ is defined as the unique pair in $\mathcal{E}$ (guaranteed to exist by Theorem 3.1) for which the associated spectrum coincides with $\sigma$ and the logarithmic coupling constants are

$$
\kappa_{\lambda}+\frac{t}{2 \lambda}, \quad \lambda \in \sigma
$$

${ }^{6}$ For example, take the topology inherited from the injection $(u, \mu) \rightarrow\left(m_{+}, m_{-}\right)$when the space of Herglotz-Nevanlinna functions is equipped with the topology of locally uniform convergence.

${ }^{7}$ We regard the space of finite Borel measures on $\mathbb{R}$ as the dual space of the Banach space $C_{0}(\mathbb{R})$. 
Of course, this definition is motivated by the well-known simple time evolution of spectral data for classical solutions of the Camassa-Holm equation, which constitutes the essence of our method of solution and is due to the underlying completely integrable structure; see [4, Section 6].

It is clear that the isospectral sets $\operatorname{Iso}(\sigma)$ are invariant under the conservative Camassa-Holm flow. Moreover, the bijection in (3.11) takes the conservative Camassa-Holm flow on Iso( $\sigma)$ to a simple linear flow on $\Lambda_{\sigma}$ whose solutions are explicitly given by (4.2). In conjunction with these facts, the trace formulas in Proposition 2.5 give rise to conserved quantities for the flow; cf. [41,57].

Proposition 4.1. The two functionals

$$
(u, \mu) \mapsto \int_{\mathbb{R}} u(x) d x, \quad(u, \mu) \mapsto \int_{\mathbb{R}} d \mu
$$

on $\mathcal{E}$ are invariant under the conservative Camassa-Holm flow.

Since the Wronskian $W$ is uniquely determined by the spectrum $\sigma$, it is invariant under the conservative Camassa-Holm flow. Therefore, we see that the functional

$$
(u, \mu) \mapsto \int_{\mathbb{R}} \rho(x) \mathrm{d} x
$$

on $\mathcal{E}$ is invariant as well (recall the notation from Remark 2.3). In particular, this shows that the property of the function $\rho$ vanishing almost everywhere (or equivalent, the Borel measure $v$ being singular with respect to the Lebesgue measure) is preserved. This case corresponds to the subclass of global conservative solutions of the Camassa-Holm equation in $[15,50]$, whereas the function $\rho$ is present for the general class of global conservative solutions of the two-component CamassaHolm system in [45].

Proposition 4.2. The conservative Camassa-Holm flow $\Phi$ is continuous.

Proof. Let $t, t_{k} \in \mathbb{R}$ for every $k \in \mathbb{N}$ such that $t_{k} \rightarrow t$ as $k \rightarrow \infty$ and suppose that the sequence $\left(u_{k}, \mu_{k}\right)$ converges to $(u, \mu)$ in $\mathcal{E}$ so that (3.14) holds as $k \rightarrow \infty$ for all functions $\chi \in C(\mathbb{R})$ such that the limit of $\chi(\lambda)$ as $|\lambda| \rightarrow \infty$ exists and is finite. In conjunction with Proposition 2.5, we infer from Corollary 3.5 that there is an $\varepsilon>0$ such that the intersections $(-\varepsilon, \varepsilon) \cap \sigma$ and $(-\varepsilon, \varepsilon) \cap \sigma_{k}$ are empty for every $k \in \mathbb{N}$. Given any continuous function $\tau$ on $\mathbb{R} \backslash\{0\}$ such that the limit of $\tau(\lambda)$ as $|\lambda| \rightarrow \infty$ exists and is finite, we choose a function $\chi_{ \pm} \in C(\mathbb{R})$ such that

$$
\chi_{ \pm}(\lambda)=\tau(\lambda) \mathrm{e}^{ \pm \frac{t}{2 \lambda}}, \quad|\lambda| \geqq \varepsilon,
$$

as well as constants $K, T \in \mathbb{R}$ such that $|\tau(\lambda)| \leqq K$ for all $\lambda \in \mathbb{R}$ with $|\lambda| \geqq \varepsilon$ and such that $\left|t_{k}\right| \leqq T$ for all $k \in \mathbb{N}$. We then may estimate 


$$
\begin{aligned}
& \left|\sum_{\lambda \in \sigma_{k}} \frac{\tau(\lambda)}{1+\lambda^{2}} \frac{1}{\lambda \gamma_{k, \lambda, \pm}^{2} \mathrm{e}^{\mp \frac{t_{k}}{2 \lambda}}}-\sum_{\lambda \in \sigma} \frac{\tau(\lambda)}{1+\lambda^{2}} \frac{1}{\lambda \gamma_{\lambda, \pm}^{2} \mathrm{e}^{\mp \frac{t}{2 \lambda}}}\right| \\
& \quad \leqq \frac{K}{\varepsilon} \mathrm{e}^{\frac{T}{\varepsilon}}\left|t_{k}-t\right| \sum_{\lambda \in \sigma_{k}} \frac{1}{1+\lambda^{2}} \frac{1}{\lambda \gamma_{k, \lambda, \pm}^{2}} \\
& \quad+\left|\sum_{\lambda \in \sigma_{k}} \frac{\chi_{ \pm}(\lambda)}{1+\lambda^{2}} \frac{1}{\lambda \gamma_{k, \lambda, \pm}^{2}}-\sum_{\lambda \in \sigma} \frac{\chi_{ \pm}(\lambda)}{1+\lambda^{2}} \frac{1}{\lambda \gamma_{\lambda, \pm}^{2}}\right|
\end{aligned}
$$

for every $k \in \mathbb{N}$. Since it follows readily from our assumptions that the right-hand side always converges to zero as $k \rightarrow \infty$, we infer that

$$
\begin{gathered}
\sum_{\lambda \in \sigma_{k}} \frac{1}{\lambda\left(1+\lambda^{2}\right)} \frac{1}{\lambda \gamma_{k, \lambda, \pm}^{2} \mathrm{e}^{\mp \frac{t_{k}}{2 \lambda}}} \rightarrow \sum_{\lambda \in \sigma} \frac{1}{\lambda\left(1+\lambda^{2}\right)} \frac{1}{\lambda \gamma_{\lambda, \pm}^{2} \mathrm{e}^{\mp \frac{t}{2 \lambda}}}, \\
\sum_{\lambda \in \sigma_{k}} \frac{\chi(\lambda)}{1+\lambda^{2}} \frac{1}{\lambda \gamma_{k, \lambda, \pm}^{2} \mathrm{e}^{\mp \frac{t_{k}}{2 \lambda}}} \rightarrow \sum_{\lambda \in \sigma} \frac{\chi(\lambda)}{1+\lambda^{2}} \frac{1}{\lambda \gamma_{\lambda, \pm}^{2} \mathrm{e}^{\mp \frac{t}{2 \lambda}}},
\end{gathered}
$$

as $k \rightarrow \infty$ for all functions $\chi \in C(\mathbb{R})$ such that the limit of $\chi(\lambda)$ as $|\lambda| \rightarrow \infty$ exists and is finite. In view of Proposition 3.4 and the definition of the flow $\Phi$, this implies that the corresponding images $\Phi^{t_{k}}\left(u_{k}, \mu_{k}\right)$ converge to $\Phi^{t}(u, \mu)$.

For the global conservative solutions of the two-component Camassa-Holm system in [45], continuity results similar to Proposition 4.2 were obtained in [45, Theorem 5.2] and [46, Theorem 6.7]. However, the topologies used in [45,46] are defined in a much more intricate and less explicit way; cf. [45, Lemma 6.4 and Lemma 6.5].

As the main result of this section, we are now going to show that the integral curve $t \mapsto \Phi^{t}\left(u_{0}, \mu_{0}\right)$ for any fixed initial data $\left(u_{0}, \mu_{0}\right) \in \mathcal{E}$ defines a weak solution of the two-component Camassa-Holm system (1.3). To this end, let us denote the pair $\Phi^{t}\left(u_{0}, \mu_{0}\right)$ with $(u(\cdot, t), \mu(\cdot, t))$ for every $t \in \mathbb{R}$, so that $u$ can be regarded as a function on $\mathbb{R} \times \mathbb{R}$. Note that the integral curve $t \mapsto \Phi^{t}\left(u_{0}, \mu_{0}\right)$ is continuous by Proposition 4.2, which guarantees that the function $u$ is at least continuous.

Theorem 4.3. The pair $(u, \mu)$ is a weak solution of the two-component CamassaHolm system (1.3) in the sense that for every test function $\varphi \in C_{\mathrm{c}}^{\infty}\left(\mathbb{R}^{2}\right)$ we have

$$
\begin{aligned}
& \int_{\mathbb{R}} \int_{\mathbb{R}} u(x, t) \varphi_{t}(x, t)+\left(\frac{u(x, t)^{2}}{2}+P(x, t)\right) \varphi_{x}(x, t) \mathrm{d} x \mathrm{~d} t=0, \\
& \int_{\mathbb{R}} \int_{\mathbb{R}} \varphi_{t}(x, t)+u(x, t) \varphi_{x}(x, t) \mathrm{d} \mu(x, t) \mathrm{d} t \\
& \quad=2 \int_{\mathbb{R}} \int_{\mathbb{R}} u(x, t)\left(\frac{u(x, t)^{2}}{2}-P(x, t)\right) \varphi_{x}(x, t) x \mathrm{~d} t,
\end{aligned}
$$


where the function $P$ is given by

$$
P(x, t)=\frac{1}{4} \int_{\mathbb{R}} \mathrm{e}^{-|x-s|} u(s, t)^{2} \mathrm{~d} s+\frac{1}{4} \int_{\mathbb{R}} \mathrm{e}^{-|x-s|} \mathrm{d} \mu(s, t), \quad x, t \in \mathbb{R} .
$$

Proof. Let us denote all the quantities corresponding to $\left(u_{0}, \mu_{0}\right)$ in an obvious way with an additional subscript. If the spectrum $\sigma_{0}$ is a finite set, then it follows from [33, Section 5] that the pair $(u, \mu)$ is a weak solution of the two-component Camassa-Holm system (1.3). Otherwise, we define a pair $\left(u_{k, 0}, \mu_{k, 0}\right) \in \mathcal{E}$ for every $k \in \mathbb{N}$ in such a way that the associated spectrum coincides with $\sigma_{0} \cap[-k, k]$ and the right/left norming constants are $\gamma_{0, \lambda, \pm}^{2}$ for every $\lambda \in \sigma_{0} \cap[-k, k]$. Since the intersection $\sigma_{0} \cap[-k, k]$ is a finite set, the corresponding pairs $\left(u_{k}, \mu_{k}\right)$ obtained from the integral curves $t \mapsto \Phi^{t}\left(u_{k, 0}, \mu_{k, 0}\right)$ satisfy

$$
\begin{aligned}
& \int_{\mathbb{R}} \int_{\mathbb{R}} u_{k}(x, t) \varphi_{t}(x, t)+\left(\frac{u_{k}(x, t)^{2}}{2}+P_{k}(x, t)\right) \varphi_{x}(x, t) \mathrm{d} x \mathrm{~d} t=0, \\
& \int_{\mathbb{R}} \int_{\mathbb{R}} \varphi_{t}(x, t)+u_{k}(x, t) \varphi_{x}(x, t) \mathrm{d} \mu_{k}(x, t) \mathrm{d} t \\
& \quad=2 \int_{\mathbb{R}} \int_{\mathbb{R}} u_{k}(x, t)\left(\frac{u_{k}(x, t)^{2}}{2}-P_{k}(x, t)\right) \varphi_{x}(x, t) \mathrm{d} x \mathrm{~d} t,
\end{aligned}
$$

for every test function $\varphi \in C_{\mathrm{c}}^{\infty}\left(\mathbb{R}^{2}\right)$, where the function $P_{k}$ is given by

$$
P_{k}(x, t)=\frac{1}{4} \int_{\mathbb{R}} \mathrm{e}^{-|x-s|} u_{k}(s, t)^{2} \mathrm{~d} s+\frac{1}{4} \int_{\mathbb{R}} \mathrm{e}^{-|x-s|} \mathrm{d} \mu_{k}(s, t), \quad x, t \in \mathbb{R} .
$$

Moreover, our definitions and Proposition 2.5 guarantee the bounds

$$
u_{k}(x, t)^{2} \leqq \mu_{k}(\mathbb{R}, t) \leqq \frac{1}{2} \sum_{\lambda \in \sigma_{0}} \frac{1}{\lambda^{2}}, \quad x, t \in \mathbb{R}, k \in \mathbb{N},
$$

and from Proposition 3.4 we infer that the functions $u_{k}(\cdot, t)$ converge to $u(\cdot, t)$ locally uniformly and that the Borel measures $\mu_{k}(\cdot, t)$ converge to $\mu(\cdot, t)$ in the weak* topology (to conclude this, we also used that the sequence $\mu_{k}(\mathbb{R}, t)$ is bounded) for every fixed $t \in \mathbb{R}$. In particular, this shows that the sequence of functions $P_{k}$ is bounded as well and converges to $P$ at least pointwise. Finally, upon passing to the limit $k \rightarrow \infty$ in (4.8) and (4.9), we infer that the pair $(u, \mu)$ is a weak solution of the two-component Camassa-Holm system (1.3).

When the Borel measure $v_{0}$ corresponding to the pair $\left(u_{0}, \mu_{0}\right)$ vanishes identically and the distribution $\omega_{0}$ is non-negative/non-positive, then this property is preserved by the conservative Camassa-Holm flow in view of Proposition 2.7; cf. [20, Corollary 3.3]. In this case, the function $u$ is a weak solution of the CamassaHolm equation (1.1) in the sense that for every test function $\varphi \in C_{\mathrm{c}}^{\infty}\left(\mathbb{R}^{2}\right)$ we have

$$
\int_{\mathbb{R}} \int_{\mathbb{R}} u(x, t) \varphi_{t}(x, t)+\left(\frac{u(x, t)^{2}}{2}+P(x, t)\right) \varphi_{x}(x, t) \mathrm{d} x \mathrm{~d} t=0,
$$


where the function $P$ is given by

$$
P(x, t)=\frac{1}{2} \int_{\mathbb{R}} \mathrm{e}^{-|x-s|} u(s, t)^{2} \mathrm{~d} s+\frac{1}{4} \int_{\mathbb{R}} \mathrm{e}^{-|x-s|} u_{x}(s, t)^{2} \mathrm{~d} s, \quad x, t \in \mathbb{R} .
$$

Under these assumptions, global weak solutions in this sense have been obtained before in $[21,26]$. In the more challenging general case, existence of global weak solutions as in Theorem 4.3 has been established in $[15,45,50]$ by means of an elaborate transformation to Lagrangian coordinates.

Remark 4.4. If the spectrum $\sigma_{0}$ associated with the pair $\left(u_{0}, \mu_{0}\right)$ is a finite set, then we recover the special class of conservative multi-peakon solutions [49], which can be written down explicitly in terms of the spectral data [33]. We have seen in the proof of Theorem 4.3 that in the general case, our weak solution $(u, \mu)$ of the two-component Camassa-Holm system (1.3) can be approximated by a sequence of conservative multi-peakon solutions in a certain way; $\mathrm{cf}$. [47,48].

We conclude this section with a comment on the long-time behavior of the conservative Camassa-Holm flow. Upon employing the method introduced in [36], it is possible to show that our weak solution $(u, \mu)$ of the two-component CamassaHolm system (1.3) asymptotically splits into a (in general infinite) train of single peakons, each corresponding to an eigenvalue $\lambda \in \sigma_{0}$ of the underlying spectral problem. For classical solutions of the Camassa-Holm equation (1.1), this behavior was anticipated by McKean [63] (in accordance with numerical observations in [17]) and first proved in [37] (see also [5,33] for the multi-peakon case and [61] for a particular class of low-regularity solutions).

\section{Appendix A. The Basic Differential Equation}

Throughout this appendix, let $u$ be a real-valued function in $H_{\text {loc }}^{1}(\mathbb{R})$ and $v$ be a non-negative Borel measure on $\mathbb{R}$. We define the distribution $\omega$ in $H_{\text {loc }}^{-1}(\mathbb{R})$ by ${ }^{8}$

$$
\omega(h)=\int_{\mathbb{R}} u(x) h(x) \mathrm{d} x+\int_{\mathbb{R}} u^{\prime}(x) h^{\prime}(x) \mathrm{d} x, \quad h \in H_{\mathrm{c}}^{1}(\mathbb{R}),
$$

so that $\omega=u-u^{\prime \prime}$ formally, and consider the ordinary differential equation

$$
-f^{\prime \prime}+\frac{1}{4} f=z \omega f+z^{2} v f
$$

where $z$ is a complex spectral parameter. Of course, this differential equation has to be understood in a distributional sense in general; cf. [35,44,67].

\footnotetext{
8 The space $H_{\mathrm{c}}^{1}(\mathbb{R})$ consists of all those functions in $H^{1}(\mathbb{R})$ which have compact support.
} 
Definition A.1. A solution of (A.2) is a function $f \in H_{\mathrm{loc}}^{1}(\mathbb{R})$ such that

$$
\int_{\mathbb{R}} f^{\prime}(x) h^{\prime}(x) \mathrm{d} x+\frac{1}{4} \int_{\mathbb{R}} f(x) h(x) \mathrm{d} x=z \omega(f h)+z^{2} \int_{\mathbb{R}} f h \mathrm{~d} v
$$

for every function $h \in H_{\mathrm{c}}^{1}(\mathbb{R})$.

In order to make this notion of solution more accessible, we will first show that the differential equation (A.2) is equivalent to the first order system

$$
\left(\begin{array}{cc}
0 & 1 \\
-1 & 0
\end{array}\right) F_{ \pm}^{\prime}=\mp \frac{1}{2}\left(\begin{array}{ll}
0 & 1 \\
1 & 0
\end{array}\right) F_{ \pm}+z\left(\begin{array}{cc}
\alpha_{ \pm}^{2} & \alpha_{ \pm} \\
\alpha_{ \pm} & 1
\end{array}\right) F_{ \pm}+z\left(\begin{array}{ll}
v & 0 \\
0 & 0
\end{array}\right) F_{ \pm},
$$

where we introduced $\alpha_{ \pm}=-u^{\prime} \pm u$. Since $v$ is allowed to be a genuine Borel measure, this system has to be understood as a measure differential equation $[3,8$, $38,65]$ in general. Solutions of such an equation are not necessarily continuous but only of locally bounded variation and, in order to guarantee the unique solvability of initial value problems, one has to impose a suitable normalization. Here we will require solutions to be left-continuous, which is implicitly contained in the following definition: a solution of the system (A.4) is a function $F_{ \pm}: \mathbb{R} \rightarrow \mathbb{C}^{2}$ with locally bounded variation such that

$$
\begin{aligned}
\left.F_{ \pm}\right|_{x} ^{y}= & \mp \frac{1}{2} \int_{x}^{y}\left(\begin{array}{cc}
-1 & 0 \\
0 & 1
\end{array}\right) F_{ \pm}(s) \mathrm{d} s+z \int_{x}^{y}\left(\begin{array}{cc}
-\alpha_{ \pm}(s) & -1 \\
\alpha_{ \pm}(s)^{2} & \alpha_{ \pm}(s)
\end{array}\right) F_{ \pm}(s) \mathrm{d} s \\
& +z \int_{x}^{y}\left(\begin{array}{ll}
0 & 0 \\
1 & 0
\end{array}\right) F_{ \pm} \mathrm{d} v
\end{aligned}
$$

for all $x, y \in \mathbb{R}$. In this case, the first component of $F_{ \pm}$is clearly locally absolutely continuous and the second component is left-continuous; cf. (1.13). With this notion of solutions, initial value problems for the system (A.4) are always uniquely solvable; see, for example, [3, Section 11.8], [8, Theorem 1.1], [38, Theorem A.2].

Lemma A.2. If the function $f$ is a solution of the differential equation (A.2), then there is a unique left-continuous function $f^{[1]}$ such that

$$
f^{[1]}(x)=f^{\prime}(x)-z u^{\prime}(x) f(x)
$$

for almost all $x \in \mathbb{R}$ and the function

$$
\left(\begin{array}{c}
z f \\
-f^{[1]} \pm\left(\frac{1}{2}-z u\right) f
\end{array}\right)
$$

is a solution of the system (A.4). Conversely, if the function $F_{ \pm}$is a solution of the system (A.4), then its first component is a solution of the differential equation (A.2).

Proof. First one notes that for every $c \in \mathbb{R}$, Equation (A.3) takes the form

$$
\begin{gathered}
\int_{\mathbb{R}} h^{\prime}(x)\left(f^{\prime}(x)-z u^{\prime}(x) f(x)-\frac{1}{4} \int_{c}^{x} f(s) \mathrm{d} s+z \int_{c}^{x} u(s) f(s)+u^{\prime}(s) f^{\prime}(s) \mathrm{d} s\right. \\
\left.+z^{2} \int_{c}^{x} f \mathrm{~d} v\right) \mathrm{d} x=0
\end{gathered}
$$


upon integrating by parts. Now from this we infer that a function $f \in H_{\text {loc }}^{1}(\mathbb{R})$ is a solution of (A.2) if and only if there is a $c \in \mathbb{R}$ and a constant $d \in \mathbb{C}$ such that

$$
\begin{gathered}
f^{\prime}(x)-z u^{\prime}(x) f(x)=d+\frac{1}{4} \int_{c}^{x} f(s) \mathrm{d} s-z \int_{c}^{x} u(s) f(s)+u^{\prime}(s) f^{\prime}(s) \mathrm{d} s \\
-z^{2} \int_{c}^{x} f \mathrm{~d} v
\end{gathered}
$$

for almost all $x \in \mathbb{R}$. So if $f$ is a solution of (A.2), then this guarantees that there is a unique left-continuous function $f^{[1]}$ such that (A.6) holds for almost all $x \in \mathbb{R}$. Let us denote the second component in (A.7) with $-f^{[ \pm]}$so that

$$
f^{[ \pm]}(x)=f^{\prime}(x) \mp \frac{1}{2} f(x)+z \alpha_{ \pm}(x) f(x)
$$

for almost all $x \in \mathbb{R}$. Then it is straightforward to show that

$$
\begin{aligned}
& \pm \frac{1}{2} \int_{x}^{y} f^{[ \pm]}(s) \mathrm{d} s+z \int_{x}^{y} \alpha_{ \pm}(s)^{2} z f(s)-\alpha_{ \pm}(s) f^{[ \pm]}(s) \mathrm{d} s \\
& \quad= \pm\left.\left(\frac{1}{2}-z u\right) f\right|_{x} ^{y}-\frac{1}{4} \int_{x}^{y} f(s) \mathrm{d} s+z \int_{x}^{y} u(s) f(s)+u^{\prime}(s) f^{\prime}(s) \mathrm{d} s
\end{aligned}
$$

for all $x, y \in \mathbb{R}$. In combination with (A.8), we end up with

$$
\begin{aligned}
-\left.f^{[ \pm]}\right|_{x} ^{y}= & \pm \frac{1}{2} \int_{x}^{y} f^{[ \pm]}(s) \mathrm{d} s+z \int_{x}^{y} \alpha_{ \pm}(s)^{2} z f(s)-\alpha_{ \pm}(s) f^{[ \pm]}(s) \mathrm{d} s \\
& +z \int_{x}^{y} z f \mathrm{~d} v
\end{aligned}
$$

for all $x, y \in \mathbb{R}$, which shows that (A.7) is a solution of the system (A.4).

Now suppose that $F_{ \pm}$is a solution of the system (A.4) and denote the respective components with subscripts. The first component of (A.5) shows that $F_{ \pm, 1}$ belongs to $H_{\text {loc }}^{1}(\mathbb{R})$ with

$$
z F_{ \pm, 2}(x)=-F_{ \pm, 1}^{\prime}(x) \pm \frac{1}{2} F_{ \pm, 1}(x)-z \alpha_{ \pm}(x) F_{ \pm, 1}(x)
$$

for almost all $x \in \mathbb{R}$. In combination with the second component of (A.5) this gives

$$
\begin{aligned}
F_{ \pm, 1}^{\prime}(x)= & \pm \frac{1}{2} F_{ \pm, 1}(x)-z \alpha_{ \pm}(x) F_{ \pm, 1}(x)-z F_{ \pm, 2}(c) \pm \frac{1}{2} \int_{c}^{x} z F_{ \pm, 2}(s) \mathrm{d} s \\
& -z^{2} \int_{c}^{x} \alpha_{ \pm}(s)^{2} F_{ \pm, 1}(s) \mathrm{d} s-z \int_{c}^{x} \alpha_{ \pm}(s) z F_{ \pm, 2}(s) \mathrm{d} s-z^{2} \int_{c}^{x} F_{ \pm, 1} \mathrm{~d} v
\end{aligned}
$$

for some $c \in \mathbb{R}$ and almost all $x \in \mathbb{R}$. Plugging (A.9) twice into this equation, we see that (A.8) holds with $f$ replaced by $F_{ \pm, 1}$ for some constant $d$. As noted before, this guarantees that $F_{ \pm, 1}$ is a solution of the differential equation (A.2).

The auxiliary function $f^{[1]}$ introduced in Lemma A.2 allows us to state the following basic existence and uniqueness result for the differential equation (A.2). 
Corollary A.3. For every $c \in \mathbb{R}$ and $d_{1}, d_{2} \in \mathbb{C}$ there is a unique solution $f$ of the differential equation (A.2) with the initial conditions

$$
f(c)=d_{1} \text { and } f^{[1]}(c)=d_{2} .
$$

If $d_{1}, d_{2}$ and $z$ are real, then the solution $f$ is real-valued as well.

Proof. If $z$ is zero, then the solutions of the differential equation (A.2) are given explicitly and the claim is obvious. Otherwise, it follows from unique solvability of initial value problems for the system (A.4) in conjunction with Lemma A.2.

We are now left to introduce the Wronskian $W(f, g)$ of two solutions $f, g$ of the differential equation (A.2). Although the following result is essentially a consequence of Lemma A.2 and the fact that the traces of the matrices in (A.5) vanish, we provide an independent direct proof.

Corollary A.4. For any two solutions $f$, $g$ of the differential equation (A.2) there is a unique complex number $W(f, g)$ such that

$$
W(f, g)=f(x) g^{\prime}(x)-f^{\prime}(x) g(x)
$$

for almost all $x \in \mathbb{R}$. This number is zero if and only if the functions $f$ and $g$ are linearly dependent.

Proof. First of all, note that if $f$ is a solution of the differential equation (A.2) and $h \in H_{\mathrm{loc}}^{1}(\mathbb{R})$, then (A.8) and the integration by parts formula (1.14) show that

$$
\begin{aligned}
\left.f^{[1]} h\right|_{x} ^{y}= & \int_{x}^{y} f^{\prime}(s) h^{\prime}(s) \mathrm{d} s+\frac{1}{4} \int_{x}^{y} f(s) h(s) \mathrm{d} s \\
& -z \int_{x}^{y} u(s) f(s) h(s)+u^{\prime}(s)(f h)^{\prime}(s) \mathrm{d} s-z^{2} \int_{x}^{y} f h \mathrm{~d} v
\end{aligned}
$$

for all $x, y \in \mathbb{R}$. If $g$ is another solution of the differential equation (A.2), then

$$
f(x) g^{\prime}(x)-f^{\prime}(x) g(x)=f(x) g^{[1]}(x)-f^{[1]}(x) g(x)
$$

for almost all $x \in \mathbb{R}$. Employing (A.12), one sees that the right-hand side of (A.13) is constant, which proves existence of the number $W(f, g)$. The remaining claim follows in a standard way upon utilizing the uniqueness part in Corollary A.3.

Let us mention that one can easily get rid of the constant potential term in the first order system (A.4) by scaling solutions with the matrix function

$$
\left(\begin{array}{cc}
\mathrm{e}^{\mp \frac{x}{2}} & 0 \\
0 & \mathrm{e}^{ \pm \frac{x}{2}}
\end{array}\right), \quad x \in \mathbb{R},
$$

at the cost of rescaling the remaining matrix coefficients in (A.4) as well. It is even possible to further transform (A.4) into a canonical system in standard form

$$
\left(\begin{array}{cc}
0 & 1 \\
-1 & 0
\end{array}\right) G_{ \pm}^{\prime}=z H_{ \pm} G_{ \pm},
$$

with a locally integrable, non-negative and trace normed matrix function $H_{ \pm}$. Since this is of no need for our purposes, we will not do this here but refer to [35, Proof of Theorem 6.1], where such a transformation has been employed. 
Acknowledgements. Open access funding provided by Austrian Science Fund (FWF).

Open Access This article is distributed under the terms of the Creative Commons Attribution 4.0 International License (http://creativecommons.org/licenses/by/4.0/), which permits unrestricted use, distribution, and reproduction in any medium, provided you give appropriate credit to the original author(s) and the source, provide a link to the Creative Commons license, and indicate if changes were made.

\section{References}

1. Ablowitz, M.J., Clarkson, P.A.: Solitons, Nonlinear Evolution Equations and Inverse Scattering, London Mathematical Society Lecture Note Series, vol. 149. Cambridge University Press, Cambridge, 1991

2. Ablowitz, M.J., Segur, H.: Solitons and the Inverse Scattering Transform, SIAM Studies in Applied Mathematics, vol. 4. SIAM, Philadelphia, 1981

3. Atkinson, F.V.: Discrete and Continuous Boundary Problems, Mathematics in Science and Engineering, vol. 8. Academic Press, New York, London, 1964

4. Beals, R., Sattinger, D.H., Szmigielski, J.: Acoustic scattering and the extended Korteweg-de Vries hierarchy. Adv. Math. 140(2), 190-206 (1998)

5. Beals, R., Sattinger, D.H., Szmigielski, J.: Multipeakons and the classical moment problem. Adv. Math. 154(2), 229-257 (2000)

6. Beals, R., Sattinger, D.H., Szmigielski, J.: Peakon-antipeakon interaction. J. Nonlinear Math. Phys. 8(suppl.), 23-27 (2001)

7. Bennewitz, C.: On the spectral problem associated with the Camassa-Holm equation. J. Nonlinear Math. Phys. 11(4), 422-434 (2004)

8. Bennewitz, C.: Spectral asymptotics for Sturm-Liouville equations. Proc. Lond. Math. Soc. (3) 59(2), 294-338 (1989)

9. Bennewitz, C., Brown, B.M., Weikard, R.: Inverse spectral and scattering theory for the half-line left-definite Sturm-Liouville problem. SIAM J. Math. Anal. 40(5), 2105$2131(2008 / 2009)$

10. Bennewitz, C., Brown, B.M., Weikard, R.: Scattering and inverse scattering for a left-definite Sturm-Liouville problem. J. Differ. Equ. 253(8), 2380-2419 (2012)

11. Bennewitz, C., Brown, B.M., Weikard, R.: The spectral problem for the dispersionless Camassa-Holm equation. In: Proceedings of the IWOTA, 2015 (to appear)

12. BoAs, R.P.: Entire Functions. Academic Press Inc., New York, 1954

13. Bogachev, V.I.: Measure Theory, Vols. I, II. Springer, Berlin, 2007

14. Boutet De Monvel, A., Shepelsky, D.: Riemann-Hilbert approach for the CamassaHolm equation on the line. Czech R. Math. Acad. Sci. Paris 343(10), 627-632 (2006)

15. Bressan, A., Constantin, A.: Global conservative solutions of the Camassa-Holm equation. Arch. Ration. Mech. Anal. 183(2), 215-239 (2007)

16. Camassa, R., Holm, D.: An integrable shallow water equation with peaked solitons, Phys. Rev. Lett. 71(11), 1661-1664 (1993)

17. Camassa, R., Holm, D., Hyman, J.: A new integrable shallow water equation. $A d v$. Appl. Mech. 31, 1-33 (1994)

18. Chen, M., LiU, S., Zhang, Y.: A two-component generalization of the Camassa-Holm equation and its solutions. Lett. Math. Phys. 75(1), 1-15 (2006)

19. Constantin, A.: On the scattering problem for the Camassa-Holm equation. R. Soc. Lond. Proc. Ser. A Math. Phys. Eng. Sci. 457(2008), 953-970 (2001)

20. Constantin, A., Escher, J.: Global existence and blow-up for a shallow water equation. Ann. Scuola Norm. Sup. Pisa Cl. Sci. (4) 26(2), 303-328 (1998)

21. Constantin, A., Escher, J.: Global weak solutions for a shallow water equation. Indiana Univ. Math. J. 47(4), 1527-1545, (1998)

22. Constantin, A., Escher, J.: Wave breaking for nonlinear nonlocal shallow water equations. Acta Math. 181(2), 229-243 (1998) 
23. Constantin, A., Ivanov, R.I.: On an integrable two-component Camassa-Holm shallow water system. Phys. Lett. A 372(48), 7129-7132 (2008)

24. Constantin, A., Lannes, D.: The hydrodynamical relevance of the Camassa-Holm and Degasperis-Procesi equations. Arch. Ration. Mech. Anal. 192(1), 165-186 (2009)

25. Constantin, A., McKean, H.P.: A shallow water equation on the circle. Commun. Pure Appl. Math. 52(8), 949-982 (1999)

26. Constantin, A., Molinet, L.: Global weak solutions for a shallow water equation. Commun. Math. Phys. 211(1), 45-61 (2000)

27. Constantin, A., Strauss, W.: Stability of peakons. Commun. Pure Appl. Math. 53(5), 603-610 (2000)

28. De Branges, L.: Hilbert Spaces of Entire Functions. Prentice-Hall, Inc., Englewood Cliffs, 1968

29. De Branges, L.: Some Hilbert spaces of entire functions. Trans. Am. Math. Soc. 96, 259-295 (1960)

30. De Branges, L.: Some Hilbert spaces of entire functions II. Trans. Am. Math. Soc. 99, $118-152(1961)$

31. De Branges, L.: Some Hilbert spaces of entire functions IV. Trans. Am. Math. Soc. 105, 43-83 (1962)

32. ECKhaRdT, J.: Direct and inverse spectral theory of singular left-definite SturmLiouville operators. J. Differ. Equ. 253(2), 604-634 (2012)

33. Eckhardt, J., Kostenko, A.: An isospectral problem for global conservative multipeakon solutions of the Camassa-Holm equation. Commun. Math. Phys. 329(3), 893918 (2014)

34. Eckhardt, J., Kostenko, A.: Quadratic operator pencils associated with the conservative Camassa-Holm flow. Bull. Soc. Math. France (to appear). arXiv:1406.3703

35. ECKhaRdt, J., Kostenko, A.: The inverse spectral problem for indefinite strings. Invent. Math. 204(3), 939-977, (2016)

36. EcKhardt, J., Teschl, G.: A coupling problem for entire functions and its application to the long-time asymptotics of integrable wave equations. Nonlinearity 29(3), 1036-1046 (2016)

37. EcKhardt, J., Teschl, G.: On the isospectral problem of the dispersionless CamassaHolm equation. Adv. Math. 235, 469-495 (2013)

38. ECKHARDT, J., Teschl, G.: Sturm-Liouville operators with measure-valued coefficients. J. Anal. Math. 120(1), 151-224 (2013)

39. EcKhaus, W., van HaRTEn, A.: The Inverse Scattering Transformation and the Theory of Solitons. North-Holland Mathematics Studies, vol. 50. North-Holland Publishing Co., Amsterdam, New York, 1981

40. Escher, J., Lechtenfeld, O., Yin, Z.: Well-posedness and blow-up phenomena for the 2-component Camassa-Holm equation. Discrete Contin. Dyn. Syst. 19(3), 493-513 (2007)

41. Fisher, M., SCHIFF, J.: The Camassa Holm equation: conserved quantities and the initial value problem. Phys. Lett. A 259(5), 371-376 (1999)

42. Gardner, C.S., Greene, J.M., Kruskal, M.D., Miura, R.M.: Method for solving the Korteweg-de Vries equation, Phys. Rev. Lett. 19(19), 1095-1097 (1967)

43. Gesztesy, F., Holden, H.: Real-valued algebro-geometric solutions of the CamassaHolm hierarchy. Philos. Trans. R. Soc. Lond. Ser. A Math. Phys. Eng. Sci. 366(1867), 1025-1054 (2008)

44. Gesztesy, F., Weikard, R.: Some remarks on the spectral problem underlying the Camassa-Holm hierarchy. In: Operator Theory in Harmonic and Non-Commutative Analysis, Operator Theory: Advances and Applications, vol. 240, pp. 137-188. Birkhäuser/Springer, Cham, 2014

45. Grunert, K., Holden, H., Raynaud, X.: Global solutions for the two-component Camassa-Holm system. Commun. Partial Differ. Equ. 37(12), 2245-2271 (2012) 
46. Grunert, K., Holden, H., Raynaud, X.: Lipschitz metric for the two-component Camassa-Holm system. In: Hyperbolic Problems: Theory, Numerics, Applications, AIMS on Applied Mathematics, vol. 8, pp. 193-207. AIMS, 2014

47. Holden, H., Raynaud, X.: A convergent numerical scheme for the Camassa-Holm equation based on multipeakons. Discrete Contin. Dyn. Syst. 14(3), 505-523 (2006)

48. Holden, H., Raynaud, X.: A numerical scheme based on multipeakons for conservative solutions of the Camassa-Holm equation. In: Hyperbolic Problems: Theory, Numerics, Applications, pp. 873-881. Springer, Berlin, 2008

49. Holden, H., Raynaud, X.: Global conservative multipeakon solutions of the CamassaHolm equation. J. Hyperb. Differ. Equ. 4(1), 39-64 (2007)

50. Holden, H., Raynaud, X.: Global conservative solutions of the Camassa-Holm equation-a Lagrangian point of view. Commun. Partial Differ. Equ. 32(10-12), 15111549 (2007)

51. Holm, D.D., Ivanov, R.I.: Two-component $\mathrm{CH}$ system: inverse scattering, peakons and geometry. Inverse Probl. 27(4), 045013, 19 (2011)

52. IONESCU-Kruse, D.: Variational derivation of the Camassa-Holm shallow water equation. J. Nonlinear Math. Phys. 14(3), 303-312 (2007)

53. Johnson, R.S.: Camassa-Holm, Korteweg-de Vries and related models for water waves. J. Fluid Mech. 455, 63-82 (2002)

54. Kaup, D.J.: Evolution of the scattering coefficients of the Camassa-Holm equation, for general initial data. Stud. Appl. Math. 117(2), 149-164 (2006)

55. Krein, M.G., Langer, H.: Continuation of Hermitian positive definite functions and related questions. Integr. Equ. Oper. Theory 78(1), 1-69 (2014)

56. Langer, M., Woracek, H.: A characterization of intermediate Weyl coefficients. Monatsh. Math. 135(2), 137-155 (2002)

57. Lenells, J.: Conservation laws of the Camassa-Holm equation. J. Phys. A 38(4), 869880 (2005)

58. Lenells, J. The correspondence between KdV and Camassa-Holm. Int. Math. Res. Not. 2004(71), 3797-3811 (2004)

59. Lenells, J.: Traveling wave solutions of the Camassa-Holm equation. J. Differ. Equ. 217(2), 393-430 (2005)

60. Levin, B.Ya.: Lectures on Entire Functions. Transl. Math. Mon., vol. 150. American Mathematical Society, Providence, 1996

61. Li, L.-C.: Long time behaviour for a class of low-regularity solutions of the CamassaHolm equation. Commun. Math. Phys. 285(1), 265-291 (2009)

62. McKean, H.P.: Breakdown of the Camassa-Holm equation. Commun. Pure Appl. Math. 57(3), 416-418 (2004)

63. McKean, H.P.: Fredholm determinants and the Camassa-Holm hierarchy. Commun. Pure Appl. Math. 56(5), 638-680 (2003)

64. McKean, H.P.: The Liouville correspondence between the Korteweg-de Vries and the Camassa-Holm hierarchies. Commun. Pure Appl. Math. 56(7), 998-1015 (2003)

65. Persson, J.: Fundamental theorems for linear measure differential equations. Math. Scand. 62(1), 19-43 (1988)

66. Rosenblum, M., RovnyaK, J.: Topics in Hardy Classes and Univalent Functions. Birkhäuser Verlag, Basel, 1994

67. Savchuk, A.M., ShKalikov, A.A.: Sturm-Liouville operators with distribution potentials. Trans. Moscow Math. Soc. 2003, 143-190 (2003)

68. WAhlén, E.: The interaction of peakons and antipeakons. Dyn. Contin. Discrete Impuls. Syst. Ser. A Math. Anal. 13(3-4), 465-472 (2006) 
69. Winkler, H.: Two-dimensional Hamiltonian systems. In: Operator Theory, pp. 525547. Springer, Basel, 2015. doi:10.1007/978-3-0348-0667-1_11

70. Xin, Z., ZHANG, P.: On the weak solutions to a shallow water equation. Commun. Pure Appl. Math. 53(11), 1411-1433 (2000)

\section{JONATHAN ECKHARDT}

Faculty of Mathematics, University of Vienna,

Oskar-Morgenstern-Platz 1, 1090 Wien, Austria.

e-mail: jonathan.eckhardt@univie.ac.at

http://homepage.univie.ac.at/jonathan.eckhardt/

(Received February 5, 2016 / Accepted November 14, 2016)

Published online November 25, 2016 - (C) The Author(s) (2016)

This article is published with open access at Springerlink.com 